\title{
On the Weyl transform for rotationally invariant symbols
}

\author{
Norbert Ortner $^{1} \cdot$ Peter Wagner $^{1}$ (D)
}

Received: 15 July 2019 / Revised: 15 July 2019 / Accepted: 25 September 2019 /

Published online: 12 October 2019

(c) The Author(s) 2019

\begin{abstract}
Several formulas for the eigenvalues $\lambda_{j}$ of the Weyl transforms $W_{\sigma}$ of symbols $\sigma$ given by radially symmetric distributions are derived. These yield criteria for the boundedness and the compactness, respectively, of the pseudo-differential operators $W_{\sigma}$. We investigate some examples by analyzing the asymptotic behavior of $\lambda_{j}$ for $j \rightarrow \infty$.
\end{abstract}

Keywords Weyl transform · Pseudo-differential operators · Rotationally invariant symbols

Mathematics Subject Classification 47G30 $\cdot 81 \mathrm{Q} 10 \cdot 46 \mathrm{~F} 10$

\section{Introduction and notation}

Our starting point for this study were the Theorems 24.5 and 26.1 in the monograph [20]. Therein, criteria for the boundedness and for the compactness, respectively, of Weyl transforms for rotation invariant symbols in the space of "temperate functions" $L_{-\infty}^{1}\left(\mathbb{R}^{2}\right)=\cup_{j \in \mathbb{N}_{0}}\left(1+x^{2}+y^{2}\right)^{j} \cdot L^{1}\left(\mathbb{R}_{x y}^{2}\right)$ are formulated. However, M. Wong's interesting example of the Dirac measure on the circle $\rho \mathbb{S}^{1}, \rho>0$, i.e., $\sigma=\delta(\rho-$ $\sqrt{x^{2}+y^{2}}$ ), already shows that there are symbols $\sigma$ not belonging to $L_{-\infty}^{1}\left(\mathbb{R}^{2}\right)$, but nevertheless leading to compact Weyl transforms, see [20, Thm. 28.1, p. 131].

In this paper, we slightly generalize [20, Thm. 24.5 , p. 115] to the space $\mathcal{S}_{r}^{\prime}\left(\mathbb{R}^{2}\right)$ of radially symmetric temperate distributions (see Proposition 3), and similarly [20, Thm. 26.1, p. 123] to the space $\mathcal{D}_{L^{1},-1}^{\prime}\left(\mathbb{R}^{2}\right)$ of weighted integrable distributions (see the Corollary to Proposition 4). In Proposition 5 and its corollary, we formulate the straight-forward generalization to $n$ dimensions. In the preparative Proposition 1, we

Peter Wagner
wagner@mat1.uibk.ac.at
Norbert Ortner
mathematik1@uibk.ac.at
$1 \quad$ University of Innsbruck, Technikerstr. 13, 6020 Innsbruck, Austria

Birkhäuser 
repeat some classical results and in Proposition 2, we give a constructive proof of B. Simon's theorem of the existence of $L^{q}$-functions, $q>2$, without bounded Weyl transforms, cf. [20, Sect. 13, p. 67].

In Sect. 5, we present three more advanced examples: We characterize the boundedness and the compactness of the Weyl transforms for the symbols $\sigma_{z}=\left(x^{2}+y^{2}\right)^{z}$, $\sigma_{z}=\left(x^{2}+y^{2}-\rho^{2}\right)_{+}^{z}$, and $\sigma_{p, z}=\chi\left(x^{2}+y^{2}\right)\left(x^{2}+y^{2}\right)^{z} \exp \left(\mathrm{i}\left(x^{2}+y^{2}\right)^{p}\right)$, respectively, for $z \in \mathbb{C} \backslash(-\mathbb{N}), \rho>0, p>1$ and $\chi \in \mathcal{C}^{\infty}(\mathbb{R})$ with $\chi(t)=1$ for $t \geq 2, \chi(t)=0$ for $t \leq 1$, in dependence on the parameters $z$ and $p$. (Note that the second example contains the Dirac measure on the circle as a limit case since $\lim _{z \rightarrow-1} t_{+}^{z} / \Gamma(1+z)=\delta(t)$ in $\mathcal{S}^{\prime}\left(\mathbb{R}_{t}^{1}\right)$.) In these examples, the determination of the asymptotic behavior of the eigenvalues of the Weyl transforms plays an essential rôle.

Let us introduce some notation. Besides the familiar Banach spaces $L^{p}\left(\mathbb{R}^{n}\right), 1 \leq$ $p \leq \infty$, we use the Banach spaces of integrable measures $\mathcal{M}^{1}\left(\mathbb{R}^{n}\right)$, see $[6$, p. 345], and of bounded and of compact linear operators on $L^{2}\left(\mathbb{R}^{n}\right)$, respectively, which we denote by $\mathcal{L}\left(L^{2}\left(\mathbb{R}^{n}\right)\right)$ and by $\operatorname{Com}\left(L^{2}\left(\mathbb{R}^{n}\right)\right)$, respectively, compare [11].

We employ the standard notation for the distribution spaces $\mathcal{D}^{\prime}, \mathcal{S}^{\prime}, \mathcal{E}^{\prime}$, the dual spaces of the spaces $\mathcal{D}, \mathcal{S}, \mathcal{E}$ of "test functions", of "rapidly decreasing functions" and of $C^{\infty}$ functions, respectively, see $[4,6,15] . \mathcal{S}_{r}^{\prime}\left(\mathbb{R}^{n}\right)$ denotes the closed subspace of $\mathcal{S}^{\prime}\left(\mathbb{R}^{n}\right)$ consisting of radially symmetric temperate distributions. In order to display the active variable in a distribution, say $x \in \mathbb{R}^{n}$, we use notation as $T(x)$ or $T \in \mathcal{D}^{\prime}\left(\mathbb{R}_{x}^{n}\right)$. Furthermore, we use at one instance the space $s^{\prime}\left(\mathbb{N}_{0}\right)$ of slowly increasing sequences, which is the dual of the space $s\left(\mathbb{N}_{0}\right)$ of fast decreasing sequences.

The spaces $\mathcal{D}_{L^{p}}, \mathcal{D}_{L^{p}}^{\prime}, 1 \leq p \leq \infty$, were introduced in [15, Ch. VI, Sect. 8 , p. 199], and we need, just for $p=1$ and $p=\infty$, so-called "weighted" $\mathcal{D}_{L^{p}}$ and $\mathcal{D}_{L^{p}}^{\prime}$-spaces, i.e.,

$$
\begin{aligned}
& \mathcal{D}_{L^{p}, \mu}:=\mathcal{D}_{L^{p}} \cdot\left(1+|x|^{2}\right)^{-\mu / 2}, \mathcal{D}_{L^{p}, \mu}^{\prime}:=\mathcal{D}_{L^{p}}^{\prime} \cdot\left(1+|x|^{2}\right)^{-\mu / 2}, \\
& 1 \leq p \leq \infty, \mu \in \mathbb{R}
\end{aligned}
$$

see $[7,8]$. (The first appearance of a weighted $\mathcal{D}_{L^{p}}^{\prime}$-space seems to be in $[13$, Sect. 3 , p. 7].) In Sect. 6, we also employ the spaces

$\mathcal{D}_{L^{\infty}, 1, \ldots, 1}:=\mathcal{D}_{L^{\infty}}\left(\mathbb{R}_{t}^{n}\right) \cdot \prod_{j=1}^{n}\left(1+t_{j}^{2}\right)^{-1 / 2}, \mathcal{D}_{L^{1},-1, \ldots,-1}^{\prime}:=\mathcal{D}_{L^{1}}^{\prime}\left(\mathbb{R}_{t}^{n}\right) \cdot \prod_{j=1}^{n} \sqrt{1+t_{j}^{2}}$.

For the evaluation of a distribution $T \in E^{\prime}$ on a test function $\phi \in E$ (where $E$ is a normal space of distributions, see [6, Ch. IV, Sect. 2, Def. 3, p. 319]), we use angle brackets, i.e., $\langle\phi, T\rangle$ or, more precisely ${ }_{E}\langle\phi, T\rangle_{E^{\prime}}$. More generally, if $\phi \in E \hat{\otimes} F$ and $T \in E^{\prime}$ for distribution spaces $E, F$, then ${ }_{E \hat{\otimes}}\langle\phi, T\rangle_{E^{\prime}}$ symbolizes the vector-valued scalar product $(E \hat{\otimes} F) \times E^{\prime} \rightarrow F$, see [14] for more information on vector-valued distributions. (In all tensor products of this study, both factors are complete and at least one of the factors is nuclear and hence $E \hat{\otimes}_{\pi} F=E \hat{\otimes}_{\epsilon} F$ and we simply write $E \hat{\otimes} F$.)

The Heaviside function is denoted by $Y$, see [15, p. 36], and we write $\delta_{\tau}(t) \in$ $\mathcal{D}^{\prime}\left(\mathbb{R}_{t}^{1}\right), \tau \in \mathbb{R}$, for the delta distribution with support in $\tau$, which is the derivative of 
$Y(t-\tau)$, i.e., $\left\langle\phi, \delta_{\tau}\right\rangle=\phi(\tau)$ for $\phi \in \mathcal{D}\left(\mathbb{R}^{1}\right)$. In contrast, $\delta \in \mathcal{D}^{\prime}\left(\mathbb{R}^{n}\right)$ without any subscript stands for the delta distribution at the origin. For $z \in \mathbb{C}$ with $\operatorname{Re} z>-1$, we write $t_{+}^{z}$ for the locally integrable function $Y(t) t^{z}$ and we obtain, by analytic continuation, the analytic distribution-valued function

$$
t_{+}^{z}: \mathbb{C} \backslash(-\mathbb{N}) \longrightarrow \mathcal{S}^{\prime}\left(\mathbb{R}_{t}^{1}\right): z \longmapsto t_{+}^{z},
$$

see [9, Ex. 1.4.8, p. 49]. The distribution $t_{-}^{z}$ is defined by $t_{-}^{z}=t_{+}^{z}(-t)$.

The pull-back $h^{*} T=T \circ h \in \mathcal{D}^{\prime}(\Omega)$ of a distribution $T$ in one variable $t$ with respect to a submersive $\mathcal{C}^{\infty}$ function $h: \Omega \rightarrow \mathbb{R}, \Omega \subset \mathbb{R}^{n}$ open, is defined as in [4, Equ. (7.2.4/5), p. 82] or in [9, Def. 1.2.12, p. 19], i.e.,

$$
\left\langle\phi, h^{*} T\right\rangle=\left\langle\frac{\mathrm{d}}{\mathrm{d} t}\left(\int_{\Omega} Y(t-h(x)) \phi(x) \mathrm{d} x\right), T\right\rangle, \quad \phi \in \mathcal{D}(\Omega) .
$$

We use the Fourier transform $\mathcal{F}$ in the form

$$
(\mathcal{F} \phi)(\xi):=\int_{\mathbb{R}^{n}} \mathrm{e}^{-\mathrm{i} \xi x} \phi(x) \mathrm{d} x, \quad \phi \in \mathcal{S}\left(\mathbb{R}^{n}\right),
$$

this being extended to $\mathcal{S}^{\prime}$ by continuity. For the partial Fourier transforms of a distribution $T \in \mathcal{S}^{\prime}\left(\mathbb{R}_{x y}^{m+n}\right)$ with respect to $x$ or $y$, respectively, we use the notation $\mathcal{F}_{x} T$ and $\mathcal{F}_{y} T$, respectively.

The Hermite polynomials $H_{j}$ and Hermite functions $h_{j}, h_{\alpha}$ are defined as usually:

$$
H_{j}(x)=(-1)^{j} \mathrm{e}^{x^{2}} \cdot\left(\mathrm{e}^{-x^{2}}\right)^{(j)}, \quad h_{j}(x)=\mathrm{e}^{-x^{2} / 2} H_{j}(x), \quad j \in \mathbb{N}_{0}, x \in \mathbb{R}
$$

and

$$
h_{\alpha}(x)=\prod_{j=1}^{n} h_{\alpha_{j}}\left(x_{j}\right), \quad \alpha \in \mathbb{N}_{0}^{n}, x \in \mathbb{R}^{n}
$$

see [5, Sect. 8.95], [20, Sects. 18, 19]. Similarly, the Laguerre polynomials $L_{j}^{\alpha}(x)$ of order $\alpha$ are defined by

$$
L_{j}^{\alpha}(x):=j !^{-1} x^{-\alpha} \mathrm{e}^{x} \cdot\left(\mathrm{e}^{-x} x^{\alpha+j}\right)^{(j)}, \quad x>0, \alpha>-1, j \in \mathbb{N}_{0},
$$

see [5, Sect. 8.97], [20, Sect. 20, p. 95]. We write $L_{j}(x):=L_{j}^{0}(x)$ for the Laguerre polynomials of order 0 .

\section{Review of definitions and classical results}

The Weyl transformation $W$ can be described as a partial Fourier transform composed with a linear transformation in $\mathbb{R}_{x y}^{2 n}$, to wit 


$$
W: \mathcal{S}^{\prime}\left(\mathbb{R}^{2 n}\right) \longrightarrow \mathcal{S}^{\prime}\left(\mathbb{R}^{2 n}\right): \sigma(x, y) \longmapsto W_{\sigma}(x, y)
$$

where

$$
W_{\sigma}(x, y)=(2 \pi)^{-n}\left(\mathcal{F}_{y} \sigma\right)\left(\frac{x+y}{2}, y-x\right)
$$

see [3, p. 79], [20, Equ.(6.7), p. 30]. Hence if $\sigma \in \mathcal{S}^{\prime}\left(\mathbb{R}^{2 n}\right)$, then its Weyl transform $W_{\sigma}$ is the pseudo-differential operator represented by the continuous linear mapping $W_{\sigma}: \mathcal{S}\left(\mathbb{R}^{n}\right) \rightarrow \mathcal{S}^{\prime}\left(\mathbb{R}^{n}\right)$ with the kernel $W_{\sigma}(x, y) \in \mathcal{S}^{\prime}\left(\mathbb{R}^{2 n}\right)$, i.e.,

$$
\left(W_{\sigma} \phi\right)(x)=\left\langle\phi(y), W_{\sigma}(x, y)\right\rangle \in \mathcal{S}^{\prime}\left(\mathbb{R}_{x}^{n}\right), \quad \phi \in \mathcal{S}\left(\mathbb{R}^{n}\right) .
$$

For $\sigma \in \mathcal{S}\left(\mathbb{R}^{2 n}\right)$ and $\phi \in \mathcal{S}\left(\mathbb{R}^{n}\right)$, this implies

$$
\begin{aligned}
\left(W_{\sigma} \phi\right)(x) & =(2 \pi)^{-n} \int \phi(y)\left(\mathcal{F}_{y} \sigma\right)\left(\frac{x+y}{2}, y-x\right) \mathrm{d} y \\
& =(2 \pi)^{-n} \iint \mathrm{e}^{\mathrm{i}(x-y) \eta} \phi(y) \sigma\left(\frac{x+y}{2}, \eta\right) \mathrm{d} y \mathrm{~d} \eta,
\end{aligned}
$$

compare [20, Equ. (4.2), p. 19].

In this study, we are mainly concerned with the question for which $\sigma$ the Weyl transform $W_{\sigma}$ can be extended to a bounded linear mapping $W_{\sigma}: L^{2}\left(\mathbb{R}^{n}\right) \rightarrow L^{2}\left(\mathbb{R}^{n}\right)$, i.e., $W_{\sigma} \in \mathcal{L}\left(L^{2}\left(\mathbb{R}^{n}\right)\right)$. In other words, we investigate for which $\sigma$ the norm $\left\|W_{\sigma} \phi\right\|_{2}$ can be bounded by a constant multiple of $\|\phi\|_{2}$ for $\phi \in \mathcal{S}\left(\mathbb{R}^{n}\right)$. If this condition is satisfied, we look for criteria guaranteeing that $W_{\sigma}$ is a compact operator in $L^{2}\left(\mathbb{R}^{n}\right)$, i.e., that $W_{\sigma} \in \operatorname{Com}\left(L^{2}\left(\mathbb{R}^{n}\right)\right)$.

Usually, $W$ is expressed by the "Wigner transform," which is a continuous sesquilinear mapping

$$
w: \mathcal{S}\left(\mathbb{R}^{n}\right) \times \mathcal{S}\left(\mathbb{R}^{n}\right) \longrightarrow \mathcal{S}\left(\mathbb{R}^{2 n}\right)
$$

The mappings $w$ and $W$ are connected by

$$
\left\langle\bar{\psi}, W_{\sigma} \phi\right\rangle=\langle w(\phi, \psi), \sigma\rangle, \quad \phi, \psi \in \mathcal{S}\left(\mathbb{R}^{n}\right)
$$

This implies

$$
\begin{aligned}
\left\langle\bar{\psi}, W_{\sigma} \phi\right\rangle & =(2 \pi)^{-n}\left\langle\overline{\psi(x)} \phi(y),\left(\mathcal{F}_{y} \sigma\right)\left(\frac{x+y}{2}, y-x\right)\right\rangle \\
& =(2 \pi)^{-n}\left\langle\overline{\psi\left(x-\frac{y}{2}\right)} \phi\left(x+\frac{y}{2}\right), \mathcal{F}_{y} \sigma\right\rangle \\
& =(2 \pi)^{-n}\left\langle\mathcal{F}_{y}\left(\overline{\psi\left(x-\frac{y}{2}\right)} \phi\left(x+\frac{y}{2}\right)\right), \sigma\right\rangle=\langle w(\phi, \psi), \sigma\rangle
\end{aligned}
$$

and hence

$$
(w(\phi, \psi))(x, y)=(2 \pi)^{-n} \int_{\mathbb{R}^{n}} \mathrm{e}^{-\mathrm{i} y \eta} \phi\left(x+\frac{\eta}{2}\right) \overline{\psi\left(x-\frac{\eta}{2}\right)} \mathrm{d} \eta .
$$


Note that we use for $w$ a normalization constant different from that in [20, Equ. (3.12), p. 15], and that we also changed, for clarity, the notation for the Wigner transform from $W(\phi, \psi)$ to $w(\phi, \psi)$.

Next note that the properties of $w$ mirror those of $W$. In fact, the bilinear mapping $(\phi, \psi) \mapsto w(\phi, \bar{\psi})$ extends from the tensor product $\mathcal{S}\left(\mathbb{R}^{n}\right) \otimes \mathcal{S}\left(\mathbb{R}^{n}\right)$ to its completion $\mathcal{S}\left(\mathbb{R}^{2 n}\right)$. This gives rise to the linear mapping

$$
\tilde{w}: \mathcal{S}\left(\mathbb{R}^{2 n}\right) \longrightarrow \mathcal{S}\left(\mathbb{R}^{2 n}\right): \Phi(x, y) \longmapsto(2 \pi)^{-n} \mathcal{F}_{y}\left(\Phi\left(x+\frac{y}{2}, x-\frac{y}{2}\right)\right)
$$

which trivially extends to continuous linear mappings from $L^{2}\left(\mathbb{R}^{2 n}\right)$ into itself, and from $\mathcal{S}^{\prime}\left(\mathbb{R}^{2 n}\right)$ into itself, respectively. We shall write again $w(\phi, \psi)$ for $\tilde{w}(\phi \otimes \bar{\psi})$ if $\phi, \psi$ both belong to $L^{2}\left(\mathbb{R}^{n}\right)$ or to $\mathcal{S}^{\prime}\left(\mathbb{R}^{n}\right)$, respectively.

Furthermore, from

$$
\begin{aligned}
\|w(\phi, \psi)\|_{\infty} & \leq(2 \pi)^{-n} \sup _{x \in \mathbb{R}^{n}}\left\|\phi\left(x+\frac{y}{2}\right) \overline{\psi\left(x-\frac{y}{2}\right)}\right\|_{L^{1}\left(\mathbb{R}_{y}^{n}\right)} \\
& \leq(2 \pi)^{-n}\|\phi\|_{2} \cdot\|\psi\|_{2}, \quad \phi, \psi \in L^{2}\left(\mathbb{R}^{n}\right)
\end{aligned}
$$

and the density of $\mathcal{S}\left(\mathbb{R}^{n}\right)$ in $L^{2}\left(\mathbb{R}^{n}\right)$, we conclude that

$$
w: L^{2}\left(\mathbb{R}^{n}\right) \times L^{2}\left(\mathbb{R}^{n}\right) \longrightarrow C_{0}\left(\mathbb{R}^{2 n}\right)
$$

is well-defined and continuous with respect to the supremum norm on $C_{0}\left(\mathbb{R}^{2 n}\right)$. This in turn leads to the following proposition.

Proposition 1 (a) If $\sigma \in L^{2}\left(\mathbb{R}^{2 n}\right)$, then $W_{\sigma} \in \operatorname{Com}\left(L^{2}\left(\mathbb{R}^{n}\right)\right)$;

(b) if $\sigma \in \mathcal{M}^{1}\left(\mathbb{R}^{2 n}\right)$, then $W_{\sigma} \in \mathcal{L}\left(L^{2}\left(\mathbb{R}^{n}\right)\right)$; if $\sigma \in L^{1}\left(\mathbb{R}^{2 n}\right)$, then $W_{\sigma} \in$ $\operatorname{Com}\left(L^{2}\left(\mathbb{R}^{n}\right)\right)$;

(c) if $\sigma \in \bigcup_{p \in[1,2]} L^{p}\left(\mathbb{R}^{2 n}\right)$, then $W_{\sigma} \in \operatorname{Com}\left(L^{2}\left(\mathbb{R}^{n}\right)\right)$;

(d) if $\sigma \in \bigcup_{p \in[1,2]} \mathcal{F}\left(L^{p}\left(\mathbb{R}^{2 n}\right)\right)$, then $W_{\sigma} \in \operatorname{Com}\left(L^{2}\left(\mathbb{R}^{n}\right)\right)$;

(e) if $\sigma \in \mathcal{F}\left(\mathcal{M}^{1}\left(\mathbb{R}^{2 n}\right)\right)$, then $W_{\sigma} \in \mathcal{L}\left(L^{2}\left(\mathbb{R}^{n}\right)\right)$.

Proof (a) Since $W_{\sigma}(x, y) \in L^{2}\left(\mathbb{R}^{2 n}\right)$ for $\sigma \in L^{2}\left(\mathbb{R}^{2 n}\right)$, it operates as a HilbertSchmidt operator on $L^{2}\left(\mathbb{R}^{n}\right)$. Of course, the inequality

$$
\left|\left\langle W_{\sigma} \phi, \psi\right\rangle\right|=\left|\left\langle W_{\sigma}, \psi \otimes \phi\right\rangle\right| \leq\left\|W_{\sigma}\right\|_{2} \cdot\|\phi\|_{2} \cdot\|\psi\|_{2}, \quad \phi, \psi \in \mathcal{S}\left(\mathbb{R}^{n}\right),
$$

implies that $W_{\sigma}$ can be extended to a continuous linear mapping in $L^{2}\left(\mathbb{R}^{n}\right)$, i.e., $W_{\sigma} \in \mathcal{L}\left(L^{2}\left(\mathbb{R}^{n}\right)\right)$. Moreover, Hilbert-Schmidt operators are compact since the functions in $L^{2}\left(\mathbb{R}^{n}\right) \otimes L^{2}\left(\mathbb{R}^{n}\right)$ (which lead to finite rank operators) are dense in $L^{2}\left(\mathbb{R}^{2 n}\right)$.

(b) If $\sigma \in \mathcal{M}^{1}\left(\mathbb{R}^{2 n}\right)$ and $\phi, \psi \in \mathcal{S}\left(\mathbb{R}^{n}\right)$, then the inequality in (2.1) implies

$$
\begin{aligned}
\left|\left\langle W_{\sigma} \phi, \psi\right\rangle\right| & =|\langle w(\phi, \bar{\psi}), \sigma\rangle| \\
& \leq\|\sigma\|_{\mathcal{M}^{1}} \cdot\|w(\phi, \bar{\psi})\|_{\infty} \leq(2 \pi)^{-n}\|\sigma\|_{\mathcal{M}^{1}} \cdot\|\phi\|_{2} \cdot\|\psi\|_{2}
\end{aligned}
$$


and hence $\left\|W_{\sigma} \phi\right\|_{2} \leq(2 \pi)^{-n}\|\sigma\|_{\mathcal{M}^{1}} \cdot\|\phi\|_{2}$. Therefore $W_{\sigma}$ belongs to $\mathcal{L}\left(L^{2}\left(\mathbb{R}^{n}\right)\right)$ and $\left\|W_{\sigma}\right\|_{\mathcal{L}\left(L^{2}\left(\mathbb{R}^{n}\right)\right)} \leq(2 \pi)^{-n}\|\sigma\|_{\mathcal{M}^{1}}$.

From the density of $\mathcal{S}\left(\mathbb{R}^{2 n}\right)$ in $L^{1}\left(\mathbb{R}^{2 n}\right)$ and since the linear mapping

$$
L^{1}\left(\mathbb{R}^{2 n}\right) \hookrightarrow \mathcal{M}^{1}\left(\mathbb{R}^{2 n}\right) \longrightarrow \mathcal{L}\left(L^{2}\left(\mathbb{R}^{n}\right)\right)
$$

is continuous, we also conclude that $W_{\sigma}$ is a compact operator for $\sigma \in L^{1}\left(\mathbb{R}^{2 n}\right)$. Note, however, that $L^{1}\left(\mathbb{R}^{2 n}\right)$ is closed and thus not dense in $\mathcal{M}^{1}\left(\mathbb{R}^{2 n}\right)$. Therefore $W_{\sigma}$ is not compact for general $\sigma \in \mathcal{M}^{1}\left(\mathbb{R}^{2 n}\right)$. E.g., if $\sigma=\delta$, then $\left(W_{\sigma} \phi\right)(x)=$ $\pi^{-n} \phi(-x)$ and this operator is clearly not compact.

(c) This follows from (a) and (b) on account of the inclusion

$$
\bigcup_{p \in[1,2]} L^{p}\left(\mathbb{R}^{2 n}\right) \subset L^{1}\left(\mathbb{R}^{2 n}\right)+L^{2}\left(\mathbb{R}^{2 n}\right) .
$$

(d) and (e) If $\sigma \in \mathcal{S}^{\prime}\left(\mathbb{R}^{2 n}\right)$ and $\sigma(x, y)=\tau\left(-\frac{y}{2}, \frac{x}{2}\right)$, then

$$
W_{\mathcal{F} \sigma}(x, y)=\left(\mathcal{F}_{x} \sigma\right)\left(\frac{x+y}{2}, x-y\right)=2^{n}\left(\mathcal{F}_{y} \tau\right)\left(\frac{y-x}{2}, x+y\right) .
$$

Hence $W_{\mathcal{F} \sigma} \phi=(4 \pi)^{n}\left(W_{\tau} \phi\right)(-x)$ for $\phi \in \mathcal{S}\left(\mathbb{R}^{n}\right)$ and (d), (e) follow from (b) and (c). The proof is complete.

Remark The statements (a), (c) and (d) of Proposition 1 correspond to the Theorems 11.1 and 11.3 in [20, pp. 55, 57]. In [20, Thm. 28.1, p. 131], $W_{\sigma}$ is considered for $\sigma=\delta\left(\rho-\sqrt{x^{2}+y^{2}}\right) \in \mathcal{M}^{1}\left(\mathbb{R}^{2}\right), \rho>0$. That $W_{\sigma}$ is a bounded operator on $L^{2}(\mathbb{R})$ follows from Proposition 1 (b). The compactness of $W_{\sigma}$ will be shown in Sect. 3 .

By the Hausdorff-Young inequality, $\mathcal{F}\left(L^{p}\left(\mathbb{R}^{2 n}\right)\right) \subset L^{q}\left(\mathbb{R}^{2 n}\right)$ if $1 \leq p \leq 2$ and $\frac{1}{p}+\frac{1}{q}=1$. The statement in Prop. 1 (d) asserts that $W_{\sigma} \in \mathcal{L}\left(L^{2}\left(\mathbb{R}^{n}\right)\right)$ holds for $\sigma$ belonging to the subspace $\mathcal{F}\left(L^{p}\left(\mathbb{R}^{2 n}\right)\right)$ of $L^{q}\left(\mathbb{R}^{2 n}\right), 2<q \leq \infty$. That $W_{\sigma}$ is not bounded on $L^{2}\left(\mathbb{R}^{n}\right)$ for general $\sigma$ in $L^{q}\left(\mathbb{R}^{2 n}\right), 2<q \leq \infty$, is easily seen if $q=\infty$. In fact the bounded function $\sigma=\mathrm{e}^{2 \mathrm{i} x y}$ yields $W_{\sigma}=2^{-n} \delta \otimes 1$ which clearly does not belong to $\mathcal{L}\left(L^{2}\left(\mathbb{R}^{n}\right)\right)$, compare [3, p. 92, (iii)], [20, Thm. 12.4, p. 61].

The case $2<q<\infty$ is trickier; it was settled in [16]. The detailed elaboration in [20, Sect. 13, p. 63] consists in an indirect proof relying on the uniform boundedness principle. We shall give a constructive proof in Proposition 2 below by means of an explicit example of $\sigma \in \bigcap_{q \in[2, \infty]} L^{q}\left(\mathbb{R}^{2}\right)$ such that $W_{\sigma} \notin \mathcal{L}\left(L^{2}(\mathbb{R})\right.$ ). (The $n$ dimensional case follows therefrom by tensoring.)

Proposition 2 The function

$$
\sigma(x, y)=\mathrm{e}^{2 \mathrm{i} x y} \cdot \frac{\Gamma(1-\mathrm{i} y) \mathrm{e}^{\pi y / 2}-1}{y \sqrt{1+|x|}}
$$

fulfills $\sigma \in C_{0}\left(\mathbb{R}^{2}\right) \cap \bigcap_{q>2} L^{q}\left(\mathbb{R}^{2}\right)$ and $W_{\sigma} \mathrm{e}^{-x^{2}} \notin L^{2}(\mathbb{R})$. In particular, $W_{\sigma} \notin$ $\mathcal{L}\left(L^{2}(\mathbb{R})\right)$. 
Proof Evidently, the function $(1+|x|)^{-1 / 2}$ belongs to $C_{0}(\mathbb{R}) \cap \bigcap_{q>2} L^{q}(\mathbb{R})$. The same holds true for

$$
f(x)=\frac{\Gamma(1-\mathrm{i} x) \mathrm{e}^{\pi x / 2}-1}{x}
$$

according to Stirling's formula, see [9, Ex. 1.6.8, (c), p. 91]. There it is also shown that $(\mathcal{F} f)(x)=2 \pi \mathrm{i}\left(Y(x)-\mathrm{e}^{\mathrm{i} \exp (-x)}\right)$. This implies

$$
(2 \pi)^{-1} \mathcal{F}_{y}\left(\mathrm{e}^{2 \mathrm{i} x y} f(y)\right)=\mathrm{i}\left(Y(y-2 x)-\mathrm{e}^{\mathrm{i} \exp (2 x-y)}\right)
$$

and

$$
W_{\sigma}(x, y)=\frac{\sqrt{2} \mathrm{i}}{\sqrt{2+|x+y|}} \cdot\left(Y(-x)-\mathrm{e}^{\mathrm{i}} \exp (2 x)\right)
$$

For $\phi=\mathrm{e}^{-x^{2}} \in \mathcal{S}(\mathbb{R})$, we conclude that

$$
\left(W_{\sigma} \phi\right)(x)=\sqrt{2} \mathrm{i}\left(Y(-x)-\mathrm{e}^{\mathrm{i}} \exp (2 x)\right) \cdot\left(\mathrm{e}^{-x^{2}} * \frac{1}{\sqrt{2+|x|}}\right)
$$

and hence, for $x>1$,

$$
\left|\left(W_{\sigma} \phi\right)(x)\right|=\sqrt{2} \mathrm{e}^{-x^{2}} * \frac{1}{\sqrt{2+|x|}} \geq \frac{\sqrt{\log 2}}{\sqrt{2} \sqrt{2+x}} .
$$

This shows that $W_{\sigma} \phi$ is not square integrable and thus $W_{\sigma} \notin \mathcal{L}\left(L^{2}(\mathbb{R})\right)$. The proof is complete.

Remark The known asymptotic of the $p$-norm (for $1 \leq p<2$ ) of the Laguerre functions $L_{j}(2 t) \mathrm{e}^{-t}$, see [17, Lemma 1.5.4, p. 28], implies, due to Proposition 3 below and via the uniform boundedness principle, that $\sigma \in L^{q}\left(\mathbb{R}^{2}\right)$ (for $2<q \leq \infty$ ) could even be chosen radially symmetric and such that $W_{\sigma}$ is unbounded on $L^{\overline{2}}(\mathbb{R})$. We are not aware, however, of an explicit construction for such a function $\sigma$.

\section{Rotationally invariant symbols}

We assume first that $n=1$ and we postpone the straight-forward generalization to higher dimensions $n$ to Sect. 6. We hence consider radially symmetric symbols $\sigma \in \mathcal{S}^{\prime}\left(\mathbb{R}^{2}\right)$ and we aim at establishing criteria for the boundedness or compactness, respectively, of the operators $W_{\sigma}$ on $L^{2}(\mathbb{R})$.

For motivation, let us first describe the two lines of reasoning in [20]: In [20, Sect. 24], $\sigma(x, y)$ is a temperate radial function and it is shown that the Hermite functions $h_{j} \in \mathcal{S}(\mathbb{R})$ (for the notation see Sect. 1) are eigenfunctions of $W_{\sigma}$, i.e., $W_{\sigma} h_{j}=\lambda_{j} h_{j}$, 
$\lambda_{j} \in \mathbb{C}, j \in \mathbb{N}_{0}$. Therefore the mapping properties of $W_{\sigma}$ are characterized by the eigenvalues $\lambda_{j}$ and it is shown that

$$
\lambda_{j}=2(-1)^{j} \int_{0}^{\infty} \sigma(\rho, 0) L_{j}\left(2 \rho^{2}\right) \mathrm{e}^{-\rho^{2}} \rho \mathrm{d} \rho
$$

where $L_{j}$ denotes Laguerre polynomials of order 0, see [20, Thm. 24.5, p. 115, and Equ. (24.13), p. 116].

On the other hand, if $\sigma$ has the representation $\sigma=(\mathcal{F} g)\left(x^{2}+y^{2}\right)$ with $g \in$ $L^{1}(\mathbb{R}) \cup L^{2}(\mathbb{R})$, then $W_{\sigma}$ is compact according to [20, Thm. 26.1, p. 123]. We shall take up this second line of reasoning in Sect. 4 below, and we shall generalize the lastmentioned theorem in [20, p. 123] in order to give a characterization of boundedness and compactness, respectively, of $W_{\sigma}$. Thereupon, we shall analyze some illustrative examples in Sect. 5.

In this section, we slightly generalize Theorem 24.5 in [20, p. 115] (and in particular formula (3.1) for the eigenvalues $\lambda_{j}$ ) from temperate radial functions to temperate radial distributions.

Since the mapping

$$
h: \mathbb{R}^{2} \backslash\{0\} \longrightarrow(0, \infty):(x, y) \longmapsto x^{2}+y^{2}
$$

is submersive, the pullback $h^{*} S, S \in \mathcal{D}^{\prime}((0, \infty))$, is well-defined (see Sect. 1), and we obtain in fact an isomorphism

$$
\mathcal{D}^{\prime}((0, \infty)) \stackrel{\sim}{\longrightarrow} \mathcal{D}_{r}^{\prime}\left(\mathbb{R}^{2} \backslash\{0\}\right): S \longmapsto S \circ h:=h^{*} S
$$

if $\mathcal{D}_{r}^{\prime}\left(\mathbb{R}^{2} \backslash\{0\}\right)$ denotes the radially symmetric distributions on $\mathbb{R}^{2} \backslash\{0\}$.

Analogously to [12], see also [18, Sect. 3.10, p. 249], the map in (3.3) yields the following isomorphism:

$$
\Phi: \mathcal{S}^{\prime}([0, \infty)) \stackrel{\sim}{\longrightarrow} \mathcal{S}_{r}^{\prime}\left(\mathbb{R}^{2}\right): S \longmapsto\left(\phi \mapsto \frac{1}{2}\left\langle\int_{\mathbb{S} 1} \phi(\sqrt{t} \omega) \mathrm{d} \omega, S_{t}\right\rangle\right)
$$

Here $\mathcal{S}^{\prime}([0, \infty))$ is the dual of $\mathcal{S}([0, \infty))=\left\{\left.\phi\right|_{[0, \infty)}: \phi \in \mathcal{S}(\mathbb{R})\right\}$ equipped with the seminorms

$$
\|\phi\|_{k, l}=\left\|(1+x)^{k} \phi^{(l)}(x)\right\|_{L^{\infty}([0, \infty))}, \quad k, l \in \mathbb{N}_{0},
$$

and the proof is based on the fact that $\int_{\mathbb{S}^{1}} \phi(\sqrt{t} \omega) \mathrm{d} \omega \in \mathcal{S}([0, \infty))$ for $\phi \in \mathcal{S}\left(\mathbb{R}^{2}\right)$.

Furthermore, we have an isomorphism

$$
\mathcal{S}^{\prime}([0, \infty)) \stackrel{\sim}{\longrightarrow}\left\{S \in \mathcal{S}^{\prime}(\mathbb{R}) ; \operatorname{supp} S \subset[0, \infty)\right\}: S \longmapsto\left(\phi \mapsto\left\langle\left.\phi\right|_{[0, \infty)}, S\right\rangle\right)
$$

E.g., the isomorphism $\Phi$ in (3.4) yields

$$
\Phi(\delta)=\pi \delta \in \mathcal{S}_{r}^{\prime}\left(\mathbb{R}^{2}\right), \quad \Phi\left(\delta^{\prime}\right)=-\frac{\pi}{4} \Delta \delta \in \mathcal{S}_{r}^{\prime}\left(\mathbb{R}^{2}\right) .
$$


In this setting, the following holds.

Proposition 3 Let $h_{j}$ and $L_{j}, j \in \mathbb{N}_{0}$, denote the Hermite functions and Laguerre polynomials, respectively, see Sect. 1, and let $\Phi$ be as in (3.4).

If $S \in \mathcal{S}^{\prime}([0, \infty))$ and $\sigma=\Phi(S) \in \mathcal{S}_{r}^{\prime}\left(\mathbb{R}^{2}\right)$, then $W_{\sigma} h_{j}=\lambda_{j} h_{j}$ where

$$
\lambda_{j}=(-1)^{j} \cdot \mathcal{S}([0, \infty))\left\langle L_{j}(2 t) \mathrm{e}^{-t}, S\right\rangle_{\mathcal{S}^{\prime}([0, \infty))}, \quad j \in \mathbb{N}_{0}
$$

In particular, $W_{\sigma} \in \mathcal{L}\left(L^{2}(\mathbb{R})\right)$ if and only if the sequence $\left(\lambda_{j}\right)_{j \in \mathbb{N}_{0}}$ is bounded, and $W_{\sigma} \in \operatorname{Com}\left(L^{2}(\mathbb{R})\right)$ if and only if $\lim _{j \rightarrow 0} \lambda_{j}=0$.

Proof For a temperate function $S$, we have $\sigma=\Phi(S)=S\left(x^{2}+y^{2}\right)$ and formula (3.1) from [20, Sect. 24] implies $W_{\sigma} h_{j}=\lambda_{j} h_{j}$ with

$$
\begin{aligned}
\lambda_{j} & =2(-1)^{j} \int_{0}^{\infty} S\left(\rho^{2}\right) L_{j}\left(2 \rho^{2}\right) \mathrm{e}^{-\rho^{2}} \rho \mathrm{d} \rho \\
& =(-1)^{j} \int_{0}^{\infty} S(t) L_{j}(2 t) \mathrm{e}^{-t} \mathrm{~d} t=(-1)^{j}\left\langle L_{j}(2 t) \mathrm{e}^{-t}, S\right\rangle, \quad j \in \mathbb{N}_{0} .
\end{aligned}
$$

Therefore, formula (3.5) holds for general $S \in \mathcal{S}^{\prime}([0, \infty)$ ) by the density of temperate functions in $\mathcal{S}^{\prime}([0, \infty))$.

For the remaining assertions in Proposition 3, we follow the reasoning in [20, Sect. 24]. We note that $e_{j}:=\left\|h_{j}\right\|_{2}^{-1} h_{j}, j \in \mathbb{N}_{0}$, yields an orthonormal basis in $L^{2}(\mathbb{R})$ such that $\sum_{j=0}^{\infty}\left\langle\phi, \overline{e_{j}}\right\rangle e_{j}$ converges not just in $L^{2}(\mathbb{R})$ but also in $\mathcal{S}(\mathbb{R})$ to $\phi$ for $\phi \in \mathcal{S}(\mathbb{R})$, compare [15, Ch. VII, Sect. 7, Ex. 7, pp. 260, 262] or [20, Thm. 24.2, p. 114]. Clearly, if the sequence $\left(\lambda_{j}\right)_{j \in \mathbb{N}_{0}}$ is not bounded, then $W_{\sigma}$ cannot be extended to a bounded operator on $L^{2}(\mathbb{R})$. On the other hand, if the sequence $\left(\lambda_{j}\right)_{j \in \mathbb{N}_{0}}$ is bounded, then the continuity of $W_{\sigma}$ on $\mathcal{S}(\mathbb{R})$ implies

$$
W_{\sigma} \phi=\sum_{j=0}^{\infty} \lambda_{j}\left\langle\phi, \overline{e_{j}}\right\rangle e_{j}, \quad \phi \in \mathcal{S}(\mathbb{R})
$$

and thus $\left\|W_{\sigma} \phi\right\|_{2} \leq\|\phi\|_{2} \cdot \sup _{j \in \mathbb{N}_{0}}\left|\lambda_{j}\right|, W_{\sigma} \in \mathcal{L}\left(L^{2}(\mathbb{R})\right)$ and $\left\|W_{\sigma}\right\|_{\mathcal{L}\left(L^{2}(\mathbb{R})\right)}=$ $\sup _{j \in \mathbb{N}_{0}}\left|\lambda_{j}\right|$. Finally, it is well-known that a bounded diagonal operator is compact if and only if the series of its eigenvalues converges to 0 . This completes the proof.

Examples For the measure $\sigma=\delta\left(\rho-\sqrt{x^{2}+y^{2}}\right)=\delta\left(\rho^{2}-x^{2}-y^{2}\right) \cdot 2 \rho, \rho>0$, we have to set $S=2 \rho \delta_{\rho^{2}}$ and we obtain $\lambda_{j}=2(-1)^{j} \rho L_{j}\left(2 \rho^{2}\right) \mathrm{e}^{-\rho^{2}}$. Due to the asymptotic behavior of the Laguerre polynomials, see [5, Equ. 8.978.3],

$$
\lambda_{j}=\frac{2(-1)^{j} \sqrt{\rho}}{\sqrt{\pi} \sqrt[4]{2 j}} \cdot \cos \left(2 \rho \sqrt{2 j}-\frac{\pi}{4}\right)+O\left(j^{-3 / 4}\right), \quad j \rightarrow \infty,
$$

and hence $W_{\sigma} \in \operatorname{Com}\left(L^{2}(\mathbb{R})\right)$ by Proposition 3, see also [20, Thm. 28.1, p. 131]. 
Instead, if $\sigma=\delta$, then $S=\frac{1}{\pi} \delta$ and $\lambda_{j}=\frac{1}{\pi}(-1)^{j}$ in accordance with $\left(W_{\sigma} \phi\right)(x)=$ $\frac{1}{\pi} \phi(-x)$. Hence $W_{\delta} \in \mathcal{L}\left(L^{2}(\mathbb{R})\right) \backslash \operatorname{Com}\left(L^{2}(\mathbb{R})\right)$. Similarly, for $\sigma=\Delta \delta$, we have $S=-\frac{4}{\pi} \delta^{\prime}$ and $\lambda_{j}=(-1)^{j+1} \frac{4}{\pi}(2 j+1)$ and $W_{\Delta \delta} \notin \mathcal{L}\left(L^{2}(\mathbb{R})\right)$.

Also note that the orthogonality relation

$$
\int_{0}^{\infty} L_{j}(x) L_{k}(x) \mathrm{e}^{-x} \mathrm{~d} x= \begin{cases}0: & j \neq k \\ 1: & j=k\end{cases}
$$

see [5, Equ. 7.414.3], [20, Prop. 20.1, p. 96], implies, as a consequence of formula (3.5), that, if $\left(\lambda_{j}\right) \in s^{\prime}\left(\mathbb{N}_{0}\right)$, then the symbol

$$
\sigma(x, y)=2 \mathrm{e}^{-x^{2}-y^{2}} \sum_{j=0}^{\infty}(-1)^{j} \lambda_{j} L_{j}\left(2 x^{2}+2 y^{2}\right)
$$

(where the sum converges in $\mathcal{S}^{\prime}\left(\mathbb{R}^{2}\right)$ ) has the Weyl transform with eigenvalues $\lambda_{j}$. In particular, if $\left(\lambda_{j}\right)_{j \in \mathbb{N}_{0}}$ is a bounded sequence, then $W_{\sigma}(x, y)=\sum_{j=0}^{\infty} \lambda_{j} e_{j}(x) e_{j}(y) \in$ $\mathcal{L}\left(L^{2}(\mathbb{R})\right)$. As in the proof above, we have set here $e_{j}=\left\|h_{j}\right\|_{2}^{-1} h_{j}, j \in \mathbb{N}_{0}$.

The series expansions in [5, Equs. 8.975, 8.976] and in [1, Equ. 5.11.1] therefore provide us with several examples, e.g.,

$$
\begin{aligned}
& \sigma(x, y)=2 J_{0}\left(2^{3 / 2} \sqrt{-z\left(x^{2}+y^{2}\right)}\right) \mathrm{e}^{-z-x^{2}-y^{2}} \in \mathcal{S}\left(\mathbb{R}^{2}\right) \Longrightarrow \lambda_{j}=\frac{z^{j}}{j !}, \quad z \in \mathbb{C} ; \\
& \sigma(x, y)=-2 \mathrm{e}^{x^{2}+y^{2}} \operatorname{Ei}\left(-2 x^{2}-2 y^{2}\right) \in L^{2}\left(\mathbb{R}^{2}\right) \Longrightarrow \lambda_{j}=\frac{(-1)^{j}}{j+1} \\
& \sigma(x, y)=K_{0}\left(x^{2}+y^{2}\right) \in L^{2}\left(\mathbb{R}^{2}\right) \Longrightarrow \lambda_{j}=\frac{(-1)^{j}}{2 j+1}
\end{aligned}
$$

Note that the Weyl transforms $W_{\sigma}$ in the last three examples are even Hilbert-Schmidt operators.

\section{Symbols represented by radial Fourier transformation}

Since the mapping $h(x, y)=x^{2}+y^{2}$ (compare (3.2)) is not submersive at 0 , some care has to be exercised when composing a distribution $S \in \mathcal{D}^{\prime}(\mathbb{R})$ with $h$. In Sect. 3, we took recourse to the space $\mathcal{S}^{\prime}([0, \infty))$, here we shall consider $S=\mathcal{F} T$ for $T \in$ $\mathcal{D}_{L^{1},-1}^{\prime}(\mathbb{R})$. Then we can define $\sigma=h^{*} S=(\mathcal{F} T)\left(x^{2}+y^{2}\right) \in \mathcal{S}^{\prime}\left(\mathbb{R}_{x y}^{2}\right)$ by the formula

$$
\langle\phi, \sigma\rangle={\mathcal{D}_{L^{1},-1}^{\prime}}_{\mathbb{R}) \otimes \mathcal{S}\left(\mathbb{R}^{2}\right)}\left\langle T \otimes \phi, \mathrm{e}^{-\mathrm{i} t\left(x^{2}+y^{2}\right)}\right\rangle_{\mathcal{D}_{L^{\infty}, 1}(\mathbb{R}) \hat{\otimes} \mathcal{S}^{\prime}\left(\mathbb{R}^{2}\right)}, \quad \phi \in \mathcal{S}\left(\mathbb{R}^{2}\right),
$$


since $\mathrm{e}^{-\mathrm{i} t\left(x^{2}+y^{2}\right)} \in \mathcal{D}_{L^{\infty}, 1}\left(\mathbb{R}_{t}^{1}\right) \hat{\otimes} \mathcal{S}^{\prime}\left(\mathbb{R}_{x y}^{2}\right)$ by [10, Lemma 2.2, p. 266]. As a shorthand for (4.1), we write $\sigma=(\mathcal{F} T)\left(x^{2}+y^{2}\right)=\mathcal{D}_{L^{1},-1}^{\prime}\left\langle T(t), \mathrm{e}^{-i t\left(x^{2}+y^{2}\right)}\right\rangle_{\mathcal{D}_{L^{\infty}, 1} \hat{\otimes} \mathcal{S}^{\prime}\left(\mathbb{R}^{2}\right)}$, see the notation in Sect. 1. Of course, in $\mathbb{R}^{2} \backslash\{0\}$, the distribution $\sigma$ coincides with the pullback $h^{*} S$ defined in (3.3).

Proposition 4 Let $T \in \mathcal{D}_{L^{1},-1}^{\prime}(\mathbb{R})$ and set

$$
\sigma=(\mathcal{F} T)\left(x^{2}+y^{2}\right)={\mathcal{D}_{L^{1},-1}^{\prime}}\left\langle T(t), \mathrm{e}^{-\mathrm{i} t\left(x^{2}+y^{2}\right)}\right\rangle_{\mathcal{D}_{L^{\infty}, 1} \hat{\otimes} \mathcal{S}^{\prime}\left(\mathbb{R}^{2}\right)} \in \mathcal{S}^{\prime}\left(\mathbb{R}^{2}\right) .
$$

Let $h_{j}$ denote the Hermite functions as in Sect. 1. Then $W_{\sigma} h_{j}=\lambda_{j} h_{j}$ where

$$
\lambda_{j}=\mathcal{D}_{L^{\infty}, 1}\left(\frac{(1-\mathrm{i} t)^{j}}{(1+\mathrm{i} t)^{j+1}}, T\right\rangle_{\mathcal{D}_{L^{1},-1}^{\prime}}, \quad j \in \mathbb{N}_{0} .
$$

Furthermore,

$$
W_{\sigma}(x, y)=\mathcal{D}_{L^{1},-1}^{\prime}\left\langle T(t), \frac{1}{\sqrt{4 \pi \mathrm{i} t}} \mathrm{e}^{\left.-\mathrm{i} t(x+y)^{2} / 4+\mathrm{i}(x-y)^{2} /(4 t)\right)}\right\rangle_{\mathcal{D}_{L^{\infty}, 1} \hat{\otimes} \mathcal{S}^{\prime}\left(\mathbb{R}^{2}\right)} \in \mathcal{S}^{\prime}\left(\mathbb{R}^{2}\right)
$$

In particular, $W_{\sigma} \in \mathcal{L}\left(L^{2}(\mathbb{R})\right)$ if and only if the sequence $\left(\lambda_{j}\right)_{j \in \mathbb{N}_{0}}$ is bounded, and $W_{\sigma} \in \operatorname{Com}\left(L^{2}(\mathbb{R})\right)$ if and only if $\lim _{j \rightarrow 0} \lambda_{j}=0$.

Proof Under somewhat stronger assumptions on $\sigma$, formula (4.2) for the eigenvalues $\lambda_{j}$ was already deduced in $[20$, Sects. 25, 26] from (3.5) by Fourier transformation. We prefer to give a direct, independent proof of (4.2), which in turn would then also yield (3.5) by the residue theorem.

If $t \neq 0$ and $\sigma_{t}(x, y)=\mathrm{e}^{-\mathrm{i} t\left(x^{2}+y^{2}\right)}$, then

$$
W_{\sigma_{t}}(x, y)=\frac{1}{\sqrt{4 \pi \mathrm{i} t}} \exp \left(-\mathrm{i} \alpha(t)\left(x^{2}+y^{2}\right)-2 \mathrm{i} \beta(t) x y\right)
$$

for $\alpha(t)=\frac{1}{4}\left(t-t^{-1}\right), \beta(t)=\frac{1}{4}\left(t+t^{-1}\right)$. Since the Weyl transformation

$$
W: \mathcal{S}^{\prime}\left(\mathbb{R}^{2}\right) \longrightarrow \mathcal{S}^{\prime}\left(\mathbb{R}^{2}\right): \tau \longmapsto W_{\tau}
$$

is an isomorphism and since $\mathrm{e}^{-\mathrm{i} t\left(x^{2}+y^{2}\right)} \in \mathcal{D}_{L^{\infty}, 1}(\mathbb{R}) \hat{\otimes} \mathcal{S}^{\prime}\left(\mathbb{R}^{2}\right)$ by [10, Lemma 2.2, p. 266], we conclude that the kernel $K(t, x, y)=W\left(\mathrm{e}^{-\mathrm{i} t\left(x^{2}+y^{2}\right)}\right)$ also belongs to $\mathcal{D}_{L^{\infty}, 1}(\mathbb{R}) \hat{\otimes} \mathcal{S}^{\prime}\left(\mathbb{R}^{2}\right)$. For $t \neq 0$, it is given by the $\mathcal{C}^{\infty}$ function in (4.3). (Of course, $K$ also depends $C^{\infty}$ on $t \in \mathbb{R}$ with values in $\mathcal{S}^{\prime}\left(\mathbb{R}^{2}\right)$ and $K(0, x, y)=W_{1}(x, y)=$ $\delta(y-x)$.) This implies that

$$
W_{\sigma}=\mathcal{D}_{L^{1},-1}^{\prime}\langle T(t), K(t, x, y)\rangle_{\mathcal{D}_{L^{\infty}, 1} \hat{\otimes} \mathcal{S}^{\prime}\left(\mathbb{R}^{2}\right)} \in \mathcal{S}^{\prime}\left(\mathbb{R}^{2}\right) .
$$


When applying $W_{\sigma}$ to a test function $\phi \in \mathcal{S}(\mathbb{R})$ we therefore conclude by means of Fubini's Theorem, see [14, Cor., p. 136], that

$$
W_{\sigma} \phi=\mathcal{D}_{L^{1},-1}^{\prime}\left\langle T(t), W_{\sigma_{t}} \phi\right\rangle_{\mathcal{D}_{L^{\infty}, 1} \hat{\otimes} \mathcal{S}^{\prime}(\mathbb{R})} \in \mathcal{S}^{\prime}(\mathbb{R})
$$

We shall show below that

$$
W_{\sigma_{t}} h_{j}=\frac{(1-\mathrm{i} t)^{j}}{(1+\mathrm{i} t)^{j+1}} \cdot h_{j}, \quad t \in \mathbb{R},
$$

and this will imply then formula (4.2). The remaining statements in Proposition 4 follow therefrom as in Proposition 3.

For $t=0$, formula (4.4) is immediate, and for $t \neq 0$, we infer from (4.3) the following:

$$
\begin{aligned}
\left(W_{\sigma_{t}} h_{j}\right)(x)= & \frac{1}{\sqrt{4 \pi \mathrm{i} t}} \int_{\mathbb{R}} h_{j}(y) \mathrm{e}^{-\mathrm{i} \alpha(t)\left(x^{2}+y^{2}\right)-2 \mathrm{i} \beta(t) x y} \mathrm{~d} y \\
= & \frac{\mathrm{e}^{-\mathrm{i} \alpha(t) x^{2}}}{\sqrt{4 \pi \mathrm{i} t}} \int_{\mathbb{R}} H_{j}(y) \exp \left(-\left(\frac{1}{2}+\mathrm{i} \alpha(t)\right) y^{2}-2 \mathrm{i} \beta(t) x y\right) \mathrm{d} y \\
= & \frac{1}{\sqrt{4 \pi \mathrm{i} t}} \int_{\mathbb{R}} H_{j}(y) \exp \left(-\left(\frac{1}{2}+\mathrm{i} \alpha(t)\right)\left[y+\frac{\mathrm{i} \beta(t) x}{\frac{1}{2}+\mathrm{i} \alpha(t)}\right]^{2}\right) \mathrm{d} y \\
& \times \exp \left(x^{2}\left[-\mathrm{i} \alpha(t)-\frac{\beta(t)^{2}}{\frac{1}{2}+\mathrm{i} \alpha(t)}\right]\right) .
\end{aligned}
$$

Due to $\alpha(t)^{2}-\beta(t)^{2}=-\frac{1}{4}$, we have $-\mathrm{i} \alpha(t)-\frac{\beta(t)^{2}}{\frac{1}{2}+\mathrm{i} \alpha(t)}=-\frac{1}{2}$. Furthermore, [5, Equ. 7.374.10] yields

$$
\begin{aligned}
\left(W_{\sigma_{t}} h_{j}\right)(x) & =\frac{\mathrm{e}^{-x^{2} / 2}}{\sqrt{4 \pi \mathrm{i} t}} \cdot \sqrt{\pi} \cdot \frac{\left(\mathrm{i} \alpha(t)-\frac{1}{2}\right)^{j / 2}}{\left(\mathrm{i} \alpha(t)+\frac{1}{2}\right)^{(j+1) / 2}} \cdot H_{j}\left(-\frac{\mathrm{i} \beta(t) x}{\frac{1}{2}+\mathrm{i} \alpha(t)} \sqrt{\frac{\mathrm{i} \alpha(t)+\frac{1}{2}}{\mathrm{i} \alpha(t)-\frac{1}{2}}}\right) \\
& =\frac{(1-\mathrm{i} t)^{j}}{(1+\mathrm{i} t)^{j+1}} \cdot h_{j}(x)
\end{aligned}
$$

due to $\mathrm{i} \alpha(t)+\frac{1}{2}=\frac{(1+\mathrm{i} t)^{2}}{4 \mathrm{i} t}$ and $\mathrm{i} \alpha(t)-\frac{1}{2}=\frac{(1-\mathrm{i} t)^{2}}{4 \mathrm{i} t}$. This concludes the proof.

Corollary For $T \in \mathcal{D}_{L^{1},-1}^{\prime}(\mathbb{R})$ let $\sigma=(\mathcal{F} T)\left(x^{2}+y^{2}\right) \in \mathcal{S}^{\prime}\left(\mathbb{R}^{2}\right)$.

(a) If $T \in \sqrt{1+t^{2}} \cdot \mathcal{M}^{1}(\mathbb{R})$, then $W_{\sigma} \in \mathcal{L}\left(L^{2}(\mathbb{R})\right)$;

(b) if $T \in \sqrt{1+t^{2}} \cdot L^{1}(\mathbb{R})$, then $W_{\sigma} \in \operatorname{Com}\left(L^{2}(\mathbb{R})\right)$;

(c) if $T \in \bigcup_{p \in[1, \infty)} L^{p}(\mathbb{R})$, then $W_{\sigma} \in \operatorname{Com}\left(L^{2}(\mathbb{R})\right)$. 
Proof (a) In fact, if $T=\sqrt{1+t^{2}} \cdot \mu$ with $\mu \in \mathcal{M}^{1}(\mathbb{R})$, then the eigenvalues $\lambda_{j}$ of $W_{\sigma}$ are bounded by $\|\mu\|_{\mathcal{M}^{1}}$ due to formula (4.2):

$$
\left|\lambda_{j}\right|=\left|\left\langle\frac{(1-\mathrm{i} t)^{j}}{(1+\mathrm{i} t)^{j+1}} \cdot \sqrt{1+t^{2}}, \mu\right\rangle\right| \leq\|\mu\|_{\mathcal{M}^{1}}
$$

since the function $(1-\mathrm{i} t)^{j} \cdot(1+\mathrm{i} t)^{-j-1} \cdot \sqrt{1+t^{2}}$ has absolute value 1 for each real $t$. Hence $\left\|W_{\sigma}\right\|_{\mathcal{L}\left(L^{2}(\mathbb{R})\right)} \leq\|\mu\|_{\mathcal{M}^{1}}$.

(b) Since $\mathcal{S}(\mathbb{R})$ is dense in $\sqrt{1+t^{2}} \cdot L^{1}(\mathbb{R})$ and since $W_{\sigma}$ is a compact operator for $T \in \mathcal{S}(\mathbb{R})$, we conclude that $W_{\sigma}$ is compact for each $T$ in $\sqrt{1+t^{2}} \cdot L^{1}(\mathbb{R})$.

(c) If $p \in[1, \infty)$ and $q \in(1, \infty]$ such that $\frac{1}{p}+\frac{1}{q}=1$, then $\left(1+t^{2}\right)^{-1 / 2} \in L^{q}(\mathbb{R})$ and hence $\left(1+t^{2}\right)^{-1 / 2} \cdot L^{p}(\mathbb{R}) \subset L^{1}(\mathbb{R})$. This implies

$$
L^{p}(\mathbb{R})=\sqrt{1+t^{2}} \cdot\left(1+t^{2}\right)^{-1 / 2} \cdot L^{p}(\mathbb{R}) \subset \sqrt{1+t^{2}} \cdot L^{1}(\mathbb{R})
$$

and therefore $W_{\sigma} \in \operatorname{Com}\left(L^{2}(\mathbb{R})\right)$ for $T \in L^{p}(\mathbb{R})$ due to (b). The proof is complete.

Remark The last corollary generalizes Theorem 26.1 in [20, p. 123]. In fact, there the compactness of $W_{\sigma}$ is proven for $T \in \cup_{p \in[1,2]} L^{p}(\mathbb{R})$ and for $T \in \cup_{p \in[1,2]} \mathcal{F}\left(L^{p}(\mathbb{R})\right)$. Note that the first assertion is contained in (c) of the corollary. The second one follows directly from Proposition 1 (c) since $T=\mathcal{F} f$ with $f \in L^{p}(\mathbb{R}), 1 \leq p \leq 2$, implies $\sigma(x, y)=2 \pi \cdot f\left(-x^{2}-y^{2}\right)$ and hence $\sigma \in L^{p}\left(\mathbb{R}^{2}\right)$.

\section{Three examples}

In the following three examples, the radially symmetric distributions $\sigma_{z}(x, y) \in$ $\mathcal{S}^{\prime}\left(\mathbb{R}_{x y}^{2}\right)$ will depend meromorphically on the complex parameter $z$, see [8] for the theoretical background. Since the mapping $W$ is linear and continuous, we infer that the eigenvalues $\lambda_{j}(z)$ are also meromorphic functions of $z$ and can be determined by analytic continuation.

Example 1 Let us start with $\sigma_{z}(x, y)=\left(x^{2}+y^{2}\right)^{z}$. This yields an analytic function

$$
\mathbb{C} \backslash(-\mathbb{N}) \longrightarrow \mathcal{S}^{\prime}\left(\mathbb{R}_{x y}^{2}\right): z \longmapsto \sigma_{z}
$$

with simple poles at $z \in-\mathbb{N}$ and residues

$$
\operatorname{Res}_{z=-1-j}=\frac{\pi \Delta^{j} \delta}{2^{2 j} j !^{2}}, \quad j \in \mathbb{N}_{0},
$$

see [8, Ex. 2.3.1, p. 41].

For $z \in \mathbb{C} \backslash \mathbb{Z}$, we have

$$
\mathcal{F}\left(\frac{t_{+}^{-z-1}}{\Gamma(-z)}\right)=(\mathrm{i} t)^{z}=\mathrm{e}^{\mathrm{i} z \pi / 2} t_{+}^{z}+\mathrm{e}^{-\mathrm{i} z \pi / 2} t_{-}^{z},
$$


see [9, Equ. (1.6.2), p. 87]. If $\operatorname{Re} z>-1$, then

$$
T_{z}:=\mathrm{e}^{-\mathrm{i} z \pi / 2} \frac{t_{+}^{-z-1}}{\Gamma(-z)} \in \mathcal{D}_{L^{1},-1}^{\prime}\left(\mathbb{R}_{t}^{1}\right)
$$

and hence $\sigma_{z}(x, y)=\left(\mathcal{F} T_{z}\right)\left(x^{2}+y^{2}\right)$ holds in the sense of formula (4.1). Therefore, formula (4.2) in Proposition 4 furnishes, if $z \in \mathbb{C} \backslash \mathbb{N}_{0}$ with $\operatorname{Re} z>-1$, the representation

$$
\lambda_{j}(z)=\mathrm{e}^{-\mathrm{i} z \pi / 2} \mathcal{D}_{L^{\infty}, 1}\left\langle\frac{(1-\mathrm{i} t)^{j}}{(1+\mathrm{i} t)^{j+1}}, \frac{t_{+}^{-z-1}}{\Gamma(-z)}\right\rangle_{\mathcal{D}_{L^{1},-1}^{\prime}}, \quad j \in \mathbb{N}_{0},
$$

for the eigenvalues of $W_{\sigma_{z}}$.

For $-1<\operatorname{Re} z<0$, this is an absolutely convergent integral we can easily evaluate:

$$
\begin{aligned}
\lambda_{j}(z) & =\frac{\mathrm{e}^{-\mathrm{i} z \pi / 2}}{\Gamma(-z)} \cdot \int_{0}^{\infty}(1-\mathrm{i} t)^{j}(1+\mathrm{i} t)^{-j-1} t^{-z-1} \mathrm{~d} t \\
& =\frac{(-1)^{j} \mathrm{e}^{-\mathrm{i}(z+1) \pi / 2}}{\Gamma(-z)} \cdot \int_{0}^{\infty} t^{-z-1}(t-\mathrm{i})^{-j-1}(t+\mathrm{i})^{j} \mathrm{~d} t \\
& =\mathrm{e}^{-\mathrm{i} z \pi} \Gamma(1+z)_{2} F_{1}(j+1,-z ; 1 ; 2)=\Gamma(1+z)_{2} F_{1}(-j,-z ; 1 ; 2)
\end{aligned}
$$

by [5, Equs. 3.197.1 and 9.131.1]. The result in formula (5.1) holds for each $z \in$ $\mathbb{C} \backslash(-\mathbb{N})$ by analytic continuation and it shows, in particular, that $\lambda_{j}(z) / \Gamma(1+z)$ is a polynomial of degree $j$ in $z$.

For $-1<\operatorname{Re} z<0$, the function $T_{z}$ is contained in $\sqrt{1+t^{2}} \cdot L^{1}(\mathbb{R})$ and hence $W_{\sigma_{z}} \in \operatorname{Com}\left(L^{2}(\mathbb{R})\right)$ by the Corollary to Proposition 4. E.g., if $z=-\frac{1}{2}$, then [2, Equ. 7.3.8.2] yields $\lambda_{j}\left(-\frac{1}{2}\right)=0$ if $j$ is odd and $\lambda_{j}\left(-\frac{1}{2}\right)=\sqrt{\pi} 2^{-j} j !\left(\frac{j}{2}\right) !^{-2}$ if $j$ is even. By means of Stirling's formula, we infer the asymptotic expansion $\lambda_{j}\left(-\frac{1}{2}\right) \sim \sqrt{2 / j}$ for $j$ even and $j \rightarrow \infty$. Also note that [5, Equ. 9.131.1] furnishes the equation

$$
\Gamma(z) \lambda_{j}(-z)=(-1)^{j} \Gamma(1-z) \lambda_{j}(z-1), \quad j \in \mathbb{N}_{0}, z \in \mathbb{C} \backslash \mathbb{Z}
$$

which also implies that $\lambda_{j}\left(-\frac{1}{2}\right)$ must vanish if $j$ is an odd number.

For $z=0$, formula (5.1) yields $\lambda_{j}(0)=1$ in accordance with $\sigma_{0}=1$ and $W_{1}=$ $\delta(x-y)$; similarly, for $z \rightarrow-1$, we have

$$
\lim _{z \rightarrow-1} \frac{\sigma_{z}}{\Gamma(1+z)}=\operatorname{Res}_{z=-1} \sigma_{z}=\pi \delta \Longrightarrow \lim _{z \rightarrow-1} \frac{W_{\sigma_{z}}}{\Gamma(1+z)}=W_{\pi \delta}=\delta(x+y)
$$

in accordance with

$$
\operatorname{Res}_{z=-1} \lambda_{j}(z)=\lim _{z \rightarrow-1} \frac{\lambda_{j}(z)}{\Gamma(1+z)}={ }_{2} F_{1}(-j, 1 ; 1 ; 2)=(-1)^{j}, \quad j \in \mathbb{N}_{0} .
$$


Let us finally investigate the asymptotic behavior of $\lambda_{j}(z)$ for general $z$. Upon inserting the standard integral representation for the hypergeometric function, see [5, Equ. 9.111], we obtain, by analytic continuation,

$$
\lambda_{j}(z)=\mathcal{E}^{\prime}\left(\mathbb { R } _ { t } ^ { 1 } \left\langle\left\langle\frac{t_{+}^{-z-1}}{\Gamma(-z)} \cdot(1-t)_{+}^{z},(1-2 t)^{j}\right\rangle_{\mathcal{C}^{\infty}\left(\mathbb{R}_{t}^{1}\right)}, \quad j \in \mathbb{N}_{0}, \quad z \in \mathbb{C} \backslash(-\mathbb{N})\right.\right.
$$

Note that $t_{+}^{-z-1} / \Gamma(-z)$ is an entire distribution-valued function of $z$ and that $(1-t)_{+}^{z}$ is holomorphic for $z \in \mathbb{C} \backslash(-\mathbb{N})$. We then approximate $(1-2 t)^{j}$ by $(1-t)^{2 j}$ near zero and by $(-1)^{j} t^{2 j}$ near one, respectively, and employ Stirling's formula to conclude from formula (5.2) that

$$
\lambda_{j}(z) \sim \begin{cases}(2 j)^{z} & : \operatorname{Re} z>-\frac{1}{2} \\ (2 j)^{z}+(-1)^{j} \frac{\Gamma(1+z)}{\Gamma(-z)}(2 j)^{-z-1} & : \operatorname{Re} z=-\frac{1}{2}, \\ (-1)^{j} \frac{\Gamma(1+z)}{\Gamma(-z)}(2 j)^{-z-1} & : \operatorname{Re} z<-\frac{1}{2},\end{cases}
$$

for $j \rightarrow \infty$ and fixed $z \in \mathbb{C} \backslash(-\mathbb{N})$. Here the precise meaning of $a_{j} \sim b_{j}$ is $a_{j}-$ $b_{j}=o\left(j^{-1 / 2+|\operatorname{Re} z+1 / 2|}\right)$ for $j \rightarrow \infty$. (Let us mention that this asymptotic expansion could also be inferred from [19].) In particular, $W_{\sigma_{z}} \in \operatorname{Com}\left(L^{2}(\mathbb{R})\right)$ if and only if $-1<\operatorname{Re} z<0$ and $W_{\sigma_{z}} \in \mathcal{L}\left(L^{2}(\mathbb{R})\right)$ if and only if $-1 \leq \operatorname{Re} z \leq 0, z \neq-1$.

Example 2 Let us fix $\rho>0$. Since $\left(t-\rho^{2}\right)_{+}^{z} \in \mathcal{S}^{\prime}(\mathbb{R})$ depends analytically on $z \in \mathbb{C} \backslash(-\mathbb{N})$, see [9, Ex. 1.4.8, p. 49], and since the mapping $h(x, y)=x^{2}+y^{2}$ is submersive at the circle $h=\rho^{2}$, we obtain an analytic function

$$
\sigma: \mathbb{C} \backslash(-\mathbb{N}) \longrightarrow \mathcal{S}^{\prime}\left(\mathbb{R}^{2}\right): z \longmapsto \sigma_{z}(x, y)=h^{*}\left(\left(t-\rho^{2}\right)_{+}^{z}\right)=\left(x^{2}+y^{2}-\rho^{2}\right)_{+}^{z} .
$$

At $z=-k, k \in \mathbb{N}$, the function $\sigma$ has simple poles with the residues

$$
\operatorname{Res}_{z=-k} \sigma_{z}=\frac{(-1)^{k-1}}{(k-1) !} \delta^{(k-1)}\left(x^{2}+y^{2}-\rho^{2}\right)
$$

see [9, Equ. (1.4.1), p. 49].

In order to study the asymptotic behavior of the eigenvalues $\lambda_{j}(z), j \in \mathbb{N}_{0}$, of $W_{\sigma_{z}}$, we first assume that $\operatorname{Re} z>-1$. Then $\left(t-\rho^{2}\right)^{z}=\mathcal{F} T$ for

$$
T=\frac{\Gamma(1+z)}{2 \pi}(-\mathrm{i} t)^{-1-z} \mathrm{e}^{\mathrm{i} \rho^{2} t} \in \mathcal{D}_{L^{1},-1}^{\prime}\left(\mathbb{R}_{t}^{1}\right),
$$

see [9, Ex. 1.6.7, p. 86], and hence formula (4.2) in Proposition 4 yields

$$
\lambda_{j}(z)=\frac{\Gamma(1+z)}{2 \pi}\left\langle\frac{(1-\mathrm{i} t)^{j}}{(1+\mathrm{i} t)^{j+1}} \mathrm{e}^{\mathrm{i} \rho^{2} t},(-\mathrm{i} t)^{-1-z}\right\rangle, \quad j \in \mathbb{N}_{0} .
$$


For $-1<\operatorname{Re} z<0$, formula (5.3) represents $\lambda_{j}(z)$ by an absolutely convergent integral. If we use

$$
\left(\frac{1-\mathrm{i} t}{1+\mathrm{i} t}\right)^{j}=(-1)^{j} \mathrm{e}^{2 \mathrm{i} j \operatorname{arccot} t}
$$

and substitute $t=\cot x$, then we obtain

$$
\begin{aligned}
\lambda_{j}(z)= & \frac{(-1)^{j} \Gamma(1+z)}{2 \pi} \int_{-\pi / 2}^{\pi / 2} \mathrm{e}^{2 \mathrm{i} j x+\mathrm{i} \rho^{2} \cot x}\left((-\mathrm{i} \cot x)^{-1-z}+(-\mathrm{i} \cot x)^{-z}\right) \mathrm{d} x \\
= & (-1)^{j} \Gamma(1+z) \mathcal{F}_{x}^{-1}\left[Y ( \frac { \pi } { 2 } - | x | ) \mathrm { e } ^ { \mathrm { i } \rho ^ { 2 } \operatorname { c o t } x } \left((-\mathrm{i} \cot x)^{-1-z}\right.\right. \\
& \left.\left.+(-\mathrm{i} \cot x)^{-z}\right)\right](2 j)
\end{aligned}
$$

Formula (5.4) remains valid for $\mathbb{C} \backslash(-\mathbb{N})$ if the distribution $S$ in square brackets is defined by analytic continuation with respect to $z$.

Therefore the asymptotic behavior of $\lambda_{j}(z)$ is determined by the behavior of the distribution $S$ at its singular support, i.e., at the three points $\pm \frac{\pi}{2}$ and 0 . The points $\pm \frac{\pi}{2}$ yield, similarly as in Example 1, the asymptotic behavior $(2 j)^{z}$. Let us consider now the origin. For $j \rightarrow \infty$, it contributes the same asymptotic behavior as

$$
\begin{aligned}
(-1)^{j} \Gamma(1+z) & \mathcal{F}_{x}^{-1}\left[\mathrm{e}^{\mathrm{i} \rho^{2} / x}(\mathrm{i} x)^{z}\right](2 j)=\frac{(-1)^{j}}{2 \pi} \Gamma(1+z) \int_{-\infty}^{\infty} \mathrm{e}^{\mathrm{i}\left(2 j x+\rho^{2} / x\right)}(\mathrm{i} x)^{z} \mathrm{~d} x \\
& =(-1)^{j} \Gamma(1+z)\left(\frac{\rho}{\sqrt{2 j}}\right)^{1+z} J_{-1-z}(2 \rho \sqrt{2 j}) \\
& \sim \frac{(-1)^{j}}{\sqrt{\pi}} \Gamma(1+z) \rho^{1 / 2+z}(2 j)^{-z / 2-3 / 4} \cos \left(2 \rho \sqrt{2 j}+\frac{\pi z}{2}+\frac{\pi}{4}\right)
\end{aligned}
$$

according to [5, Equs. 8.421.8 and 8.451.1]. (Though the integral in the first formula line is convergent only for $-2<\operatorname{Re} z<0$, the result remains valid for $z \in \mathbb{C} \backslash(-\mathbb{N})$ by analytic continuation.) Altogether we obtain for $\lambda_{j}(z)$ the following asymptotic behavior if $j \rightarrow \infty$ and $z \in \mathbb{C} \backslash(-\mathbb{N})$ is fixed:

$\lambda_{j}(z) \sim \begin{cases}(2 j)^{z} & : \operatorname{Re} z>-\frac{1}{2}, \\ (2 j)^{z}+(-1)^{j} \frac{\Gamma(1+z)}{\sqrt{\pi}} \rho^{1 / 2+z}(2 j)^{-z / 2-3 / 4} \cos \left(2 \rho \sqrt{2 j}+\frac{\pi z}{2}+\frac{\pi}{4}\right) & : \operatorname{Re} z=-\frac{1}{2}, \\ (-1)^{j} \frac{\Gamma(1+z)}{\sqrt{\pi}} \rho^{1 / 2+z}(2 j)^{-z / 2-3 / 4} \cos \left(2 \rho \sqrt{2 j}+\frac{\pi z}{2}+\frac{\pi}{4}\right) & : \operatorname{Re} z<-\frac{1}{2} .\end{cases}$

In particular, this implies that $W_{\sigma_{z}} \in \operatorname{Com}\left(L^{2}(\mathbb{R})\right)$ if and only if $-\frac{3}{2}<\operatorname{Re} z<0$ and $W_{\sigma_{z}} \in \mathcal{L}\left(L^{2}(\mathbb{R})\right)$ if and only if $-\frac{3}{2} \leq \operatorname{Re} z \leq 0, z \neq-1$. As a control, we can consider the limit

$$
\lim _{z \rightarrow-1} \frac{\sigma_{z}}{\Gamma(1+z)}=\operatorname{Res}_{z=-1} \sigma_{z}=\delta_{\rho^{2}}\left(x^{2}+y^{2}\right)
$$

and obtain the correct asymptotic behavior, compare the example in Sect. 3. 
Example 3 Let us finally consider the symbols

$$
\sigma_{p, z}=\chi\left(x^{2}+y^{2}\right)\left(x^{2}+y^{2}\right)^{z} \exp \left(\mathrm{i}\left(x^{2}+y^{2}\right)^{p}\right), \quad z \in \mathbb{C}, p>1,
$$

if $\chi \in \mathcal{C}^{\infty}(\mathbb{R})$ with $\chi(t)=1$ for $t \geq 2, \chi(t)=0$ for $t \leq 1$. (Since $\left(x^{2}+y^{2}\right)^{z}$ has already been investigated in Example 1, we are interested here only in the contributions stemming from the behavior of $\sigma_{p, z}$ near infinity and we therefore employ the excision function $\chi$.)

Since $\sigma_{p, z}(x, y)=(\mathcal{F} T)\left(x^{2}+y^{2}\right)$ with

$$
T=\mathcal{F}^{-1}\left(\chi(t) t^{z} \mathrm{e}^{\mathrm{i} t} t^{p}\right) \in \mathcal{F}^{-1}\left(\mathcal{O}_{M}\right)=\mathcal{O}_{C}^{\prime} \subset \mathcal{D}_{L^{1},-1}^{\prime}\left(\mathbb{R}_{t}^{1}\right),
$$

we can apply formula (4.2) in Proposition 4. The distribution $T$ is again a fast oscillating $\mathcal{C}^{\infty}$ function whose behavior at infinity is readily obtained by the method of stationary phase:

$$
\begin{aligned}
& \text { If } t<0 \text { and } h(s)=s^{p}+s t, \frac{1}{p}+\frac{1}{q}=1 \text { and } h^{\prime}\left(s_{0}\right)=0 \text {, then } \\
& \qquad \begin{aligned}
T(t) & =\frac{1}{2 \pi} \int_{0}^{\infty} \chi(s) s^{z} \mathrm{e}^{\mathrm{i} h(s)} \mathrm{d} s \sim \frac{1}{2 \pi} \mathrm{e}^{\mathrm{i} h\left(s_{0}\right)} \sqrt{\frac{2 \pi \mathrm{i}}{h^{\prime \prime}\left(s_{0}\right)}} \\
& =\sqrt{\frac{\mathrm{i}}{2 \pi p(p-1)}\left(\frac{|t|}{p}\right)^{(2-p+2 z) /(2(p-1))} \exp \left(-\mathrm{i}(p-1)\left(\frac{|t|}{p}\right)^{q}\right)}
\end{aligned}
\end{aligned}
$$

for $t \rightarrow-\infty$. For $t \rightarrow \infty$, the function $T(t)$ is fast decreasing.

With the same substitution as in formula (5.4), we therefore obtain for the eigenvalues $\lambda_{j}(p, z)$ of $W_{\sigma_{p, z}}$ the following:

$$
\begin{aligned}
\lambda_{j}(p, z) & =(-1)^{j} \int_{-\pi / 2}^{\pi / 2} \mathrm{e}^{2 \mathrm{i} j x} T(\cot x) \cdot(1-\mathrm{i} \cot x) \mathrm{d} x \\
& \sim \frac{(-1)^{j} \mathrm{i}^{3 / 2} p^{-(1+2 z) /(2(p-1))}}{\sqrt{2 \pi(p-1)}} \int_{0}^{\infty}(1-\chi(x)) \mathrm{e}^{-2 \mathrm{i} j x} x^{-q / 2-z /(p-1)} \mathrm{e}^{-\mathrm{i}(p-1)(p x)^{-q}} \mathrm{~d} x
\end{aligned}
$$

(For the convergence of the last integral, we have to stipulate that $\operatorname{Re} z<\frac{3}{2} p-1$. The final result in formula (5.5) below can, as before, be extended to $z \in \mathbb{C}$ by analytic continuation. This is done by representing the integral as the Fourier transform of a distribution $S$ and by employing the equation $\left(\mathcal{F} S^{\prime}\right)(2 j)=2 i j \mathcal{F} S$.)

Upon using again the method of stationary phase with $h(x)=2 j x+(p-1)(p x)^{-q}$ and $p x_{0}=\left(2 j / p^{2}\right)^{(1-p) /(2 p-1)}$, this finally implies

$$
\begin{aligned}
\lambda_{j}(p, z) & \sim \frac{(-1)^{j} \mathrm{i}^{3 / 2} p^{-(1+2 z) /(2(p-1))}}{\sqrt{2 \pi(p-1)}} x_{0}^{-q / 2-z /(p-1)} \mathrm{e}^{-\mathrm{i} h\left(x_{0}\right)} \sqrt{\frac{2 \pi}{\mathrm{i} h^{\prime \prime}\left(x_{0}\right)}} \\
& =\frac{(-1)^{j} \mathrm{i}}{\sqrt{2 p-1}} p^{-(1+2 z) /(2 p-1)}(2 j)^{(z+1-p) /(2 p-1)} \exp \left(-\mathrm{i}(2 p-1)\left(\frac{2 j}{p^{2}}\right)^{p /(2 p-1)}\right) .
\end{aligned}
$$


From formula (5.5), we can conclude that $W_{\sigma_{p, z}}$ is bounded if and only if $\operatorname{Re} z \leq p-1$ and that $W_{\sigma_{p, z}}$ is compact if and only if $\operatorname{Re} z<p-1$.

\section{Generalization to $n$ dimensions}

Let us consider here the variables in the ordering $x_{1}, y_{1}, x_{2}, y_{2}, \ldots, x_{n}, y_{n}$. We denote by $\mathcal{S}_{p r}^{\prime}\left(\mathbb{R}^{2 n}\right)$ the set of all partially radially symmetric $\sigma \in \mathcal{S}^{\prime}\left(\mathbb{R}^{2 n}\right)$, i.e., those temperate distributions $\sigma$ that are rotationally symmetric with respect to all $\left(x_{j}, y_{j}\right)$-planes, i.e., such that

$$
\sigma\left(A_{1}\left(\begin{array}{l}
x_{1} \\
y_{1}
\end{array}\right), \ldots, A_{n}\left(\begin{array}{l}
x_{n} \\
y_{n}
\end{array}\right)\right)=\sigma
$$

for all two-dimensional rotations $A_{1}, \ldots, A_{n}$. As in Sect. 3, we obtain an isomorphism

$$
\begin{aligned}
\Phi_{p r}: \mathcal{S}^{\prime}\left([0, \infty)^{n}\right) & \longrightarrow \mathcal{S}_{p r}^{\prime}\left(\mathbb{R}^{2 n}\right) \\
S & \left.\longmapsto\left(\phi \mapsto 2^{-n} \backslash \int_{\left(\mathbb{S}^{1}\right)^{n}} \phi\left(\sqrt{t_{1}} \omega_{1}, \ldots, \sqrt{t_{n}} \omega_{n}\right) \mathrm{d} \omega, S_{t}\right\rangle\right)
\end{aligned}
$$

for $\phi \in \mathcal{S}\left(\mathbb{R}^{2 n}\right)$.

Alternatively, we can consider $\sigma=(\mathcal{F} T)\left(x_{1}^{2}+y_{1}^{2}, \ldots, x_{n}^{2}+y_{n}^{2}\right)$ for

$$
T \in \mathcal{D}_{L^{1},-1, \ldots,-1}^{\prime}=\mathcal{D}_{L^{1}}^{\prime}\left(\mathbb{R}^{n}\right) \cdot \prod_{j=1}^{n} \sqrt{1+t_{j}^{2}}
$$

As in formula (4.1), $\sigma$ is defined by

$$
\langle\phi, \sigma\rangle=\mathcal{D}_{L^{1},-1, \ldots,-1}^{\prime} \otimes \mathcal{S}\left(\mathbb{R}^{2 n)}\left\langle T \otimes \phi, \mathrm{e}^{-\mathrm{i} t_{1}\left(x_{1}^{2}+y_{1}^{2}\right)-\cdots-\mathrm{i} t_{n}\left(x_{n}^{2}+y_{n}^{2}\right)}\right\rangle_{\mathcal{D}_{L^{\infty}, 1, \ldots, 1} \hat{\otimes} \mathcal{S}^{\prime}\left(\mathbb{R}^{2 n}\right)},\right.
$$

for $\phi \in \mathcal{S}\left(\mathbb{R}^{2 n}\right)$. Then Propositions 3 and 4 generalize to the following.

Proposition 5 Let $h_{\alpha}, \alpha \in \mathbb{N}_{0}^{n}$, and $L_{j}, j \in \mathbb{N}_{0}$, denote the Hermite functions and Laguerre polynomials, respectively, see Sect. 1, and let $\Phi_{p r}$ be as in (6.1). If $S \in \mathcal{S}^{\prime}\left([0, \infty)^{n}\right)$ and $\sigma=\Phi_{p r}(S) \in \mathcal{S}_{p r}^{\prime}\left(\mathbb{R}^{2 n}\right)$, then $W_{\sigma} h_{\alpha}=\lambda_{\alpha} h_{\alpha}$ where

$$
\lambda_{\alpha}=(-1)^{|\alpha|} \cdot \mathcal{S}\left([0, \infty)^{n}\right)\left\langle\prod_{j=1}^{n} L_{\alpha_{j}}\left(2 t_{j}\right) \mathrm{e}^{-t_{j}}, S\right\rangle_{\mathcal{S}^{\prime}\left([0, \infty)^{n}\right)}, \quad \alpha \in \mathbb{N}_{0}^{n}
$$


If instead $\sigma=(\mathcal{F} T)\left(x_{1}^{2}+y_{1}^{2}, \ldots, x_{n}^{2}+y_{n}^{2}\right) \in \mathcal{S}_{p r}^{\prime}\left(\mathbb{R}^{2 n}\right)$ for $T \in \mathcal{D}_{L^{1},-1, \ldots,-1}^{\prime}\left(\mathbb{R}^{n}\right)$ according to (6.2), then $W_{\sigma} h_{\alpha}=\lambda_{\alpha} h_{\alpha}$ where

$$
\lambda_{\alpha}=\mathcal{D}_{L^{\infty}, 1, \ldots, 1}\left\langle\prod_{j=1}^{n} \frac{\left(1-\mathrm{i} t_{j}\right)^{\alpha_{j}}}{\left(1+\mathrm{i} t_{j}\right)^{\alpha_{j}+1}}, T\right\rangle_{\mathcal{D}_{L^{1},-1, \ldots,-1}^{\prime}}, \quad \alpha \in \mathbb{N}_{0}^{n}
$$

In particular, $W_{\sigma} \in \mathcal{L}\left(L^{2}\left(\mathbb{R}^{n}\right)\right)$ if and only if the sequence $\left(\lambda_{\alpha}\right)_{\alpha \in \mathbb{N}_{0}^{n}}$ is bounded, and $W_{\sigma} \in \operatorname{Com}\left(L^{2}\left(\mathbb{R}^{n}\right)\right)$ if and only if $\lim _{|\alpha| \rightarrow \infty} \lambda_{\alpha}=0$.

Proof If $\sigma_{t}(x, y)=\prod_{j=1}^{n} \mathrm{e}^{-\mathrm{i} t_{j}\left(x_{j}^{2}+y_{j}^{2}\right)}$ for $t \in \mathbb{R}^{n}$, then Proposition 4 yields

$$
W_{\sigma_{t}} h_{\alpha}=\prod_{j=1}^{n}\left(W_{\sigma_{t_{j}}} h_{\alpha_{j}}\right)\left(x_{j}\right)=\prod_{j=1}^{n} \frac{\left(1-\mathrm{i} t_{j}\right)^{\alpha_{j}}}{\left(1+\mathrm{i} t_{j}\right)^{\alpha_{j}+1}} h_{\alpha_{j}}\left(x_{j}\right)
$$

and this implies formula (6.4) as in the proof of Proposition 4.

Formula (6.3) can be deduced from formula (6.4); in fact, if $\sigma=\otimes_{j=1}^{n} \delta\left(\rho_{j}^{2}-\right.$ $\left.x_{j}^{2}-y_{j}^{2}\right)$ for $\rho_{1}>0, \ldots, \rho_{n}>0$, then $\sigma=(\mathcal{F} T)\left(x_{1}^{2}+y_{1}^{2}, \ldots, x_{n}^{2}+y_{n}^{2}\right)$ for $T=(2 \pi)^{-n} \prod_{j=1}^{n} \mathrm{e}^{\mathrm{i} t_{j} \rho_{j}^{2}}$. Though $T \notin \mathcal{D}_{L^{1},-1, \ldots,-1}^{\prime}\left(\mathbb{R}^{n}\right)$, approximation yields that formula (6.4) can be applied in the form

$$
\lambda_{\alpha}=\prod_{j=1}^{n} \frac{1}{2 \pi} \int_{-\infty}^{\infty} \mathrm{e}^{\mathrm{i} t_{j} \rho_{j}^{2}} \frac{\left(1-\mathrm{i} t_{j}\right)^{\alpha_{j}}}{\left(1+\mathrm{i} t_{j}\right)^{\alpha_{j}+1}} \mathrm{~d} t_{j}
$$

where the integrals are conditionally convergent. Eventually, formula (6.3) follows upon employing the residue theorem, see the details in the proof of the corollary below.

Alternatively, formula (6.3) follows directly from Proposition 3 for tensor products $S \in \mathcal{S}^{\prime}([0, \infty)) \otimes \cdots \otimes \mathcal{S}^{\prime}([0, \infty))$ and then must hold generally by density. This completes the proof.

Let us finally specialize to symbols $\sigma$ in the space

$$
\mathcal{S}_{r}^{\prime}\left(\mathbb{R}^{2 n}\right)=\left\{\sigma \in \mathcal{S}^{\prime}\left(\mathbb{R}^{2 n}\right) ; \sigma \text { is radially symmetric with respect to all variables }\right\}
$$

According to [12] or [18, Sect. 3.10, p. 249], the isomorphism

$$
\mathcal{D}^{\prime}((0, \infty)) \stackrel{\sim}{\rightarrow} \mathcal{D}_{r}^{\prime}\left(\mathbb{R}^{2 n} \backslash\{0\}\right): S \longmapsto\left(t^{1-n} S\right) \circ\left(|x|^{2}+|y|^{2}\right)
$$

can be extended to $\mathbb{R}^{2 n}$ in the following way:

$$
\Phi_{r}: \mathcal{S}^{\prime}([0, \infty)) \stackrel{\sim}{\rightarrow} \mathcal{S}_{r}^{\prime}\left(\mathbb{R}^{2 n}\right): S \longmapsto\left(\phi \mapsto \frac{1}{2}\left\langle\int_{\mathbb{S}^{2 n-1}} \phi(\sqrt{t} \omega) \mathrm{d} \omega, S_{t}\right\rangle\right)
$$


Furthermore, if $T \in \mathcal{D}_{L^{1},-n}^{\prime}(\mathbb{R})$, then $\sigma=(\mathcal{F} T)\left(|x|^{2}+|y|^{2}\right) \in \mathcal{S}_{r}^{\prime}\left(\mathbb{R}^{2 n}\right)$ is welldefined, see [10, Lemma 2.2, p. 266].

Corollary Let $h_{\alpha}, \alpha \in \mathbb{N}_{0}^{n}$, and $L_{j}^{n-1}, j \in \mathbb{N}_{0}$, denote the Hermite functions and Laguerre polynomials, respectively, see Sect. 1, and let $\Phi_{r}$ be as in (6.5).

If $S \in \mathcal{S}^{\prime}([0, \infty))$ and $\sigma=\Phi_{r}(S) \in \mathcal{S}_{r}^{\prime}\left(\mathbb{R}^{2 n}\right)$, then $W_{\sigma} h_{\alpha}=\lambda_{|\alpha|} h_{\alpha}$ where

$$
\lambda_{j}=\frac{(-1)^{j} j !}{(j+n-1) !} \cdot \mathcal{S}_{([0, \infty))}\left\langle L_{j}^{n-1}(2 t) \mathrm{e}^{-t}, S\right\rangle_{\mathcal{S}^{\prime}([0, \infty))}, \quad j \in \mathbb{N}_{0} .
$$

If instead $\sigma=(\mathcal{F} T)\left(|x|^{2}+|y|^{2}\right) \in \mathcal{S}_{r}^{\prime}\left(\mathbb{R}^{2 n}\right)$ for $T \in \mathcal{D}_{L^{1},-n}^{\prime}(\mathbb{R})$, then $W_{\sigma} h_{\alpha}=\lambda_{|\alpha|} h_{\alpha}$ where

$$
\lambda_{j}=\mathcal{D}_{L^{\infty}, n}\left\langle\frac{(1-\mathrm{i} t)^{j}}{(1+\mathrm{i} t)^{j+n}}, T\right\rangle_{\mathcal{D}_{L^{1},-n}^{\prime}}, \quad j \in \mathbb{N}_{0} .
$$

Proof We first prove formula (6.7). If $T \in \mathcal{D}_{L^{1},-n}^{\prime}(\mathbb{R})$, then

$$
S=\delta\left(t_{1}-t_{2}\right) \cdots \delta\left(t_{n-1}-t_{n}\right) T\left(t_{n}\right) \in \mathcal{D}_{L^{1},-1, \ldots,-1}^{\prime}\left(\mathbb{R}^{n}\right)
$$

is defined as the composition of $\delta_{\mathbb{R}^{n-1}} \otimes T$ with a non-degenerate linear mapping and

$$
(\mathcal{F} S)\left(x_{1}^{2}+y_{1}^{2}, \ldots, x_{n}^{2}+y_{n}^{2}\right)=(\mathcal{F} T)\left(|x|^{2}+|y|^{2}\right) .
$$

Therefore formula (6.4) implies formula (6.7):

$$
\lambda_{\alpha}=\left\langle\prod_{j=1}^{n} \frac{\left(1-\mathrm{i} t_{j}\right)^{\alpha_{j}}}{\left(1+\mathrm{i} t_{j}\right)^{\alpha_{j}+1}}, S\right\rangle=\left\langle\frac{(1-\mathrm{i} t)^{|\alpha|}}{(1+\mathrm{i} t)^{|\alpha|+n}}, T\right\rangle .
$$

In the same way, formula (6.6) could be inferred from formula (6.3) by induction employing [5, Equ. 7.413]. It appears to be more illuminating to deduce (6.6) from (6.7) by the residue theorem. We first observe that linear combinations of $\delta_{\rho^{2}}, \rho>0$, are dense in $\mathcal{S}^{\prime}\left([0, \infty)\right.$ ). Hence it suffices to prove (6.6) for $S=\delta_{\rho^{2}}, \rho>0$. In this case,

$$
\sigma=\Phi_{r}\left(\delta_{\rho^{2}}\right)=\rho^{2(1-n)} \delta_{\rho^{2}}\left(|x|^{2}+|y|^{2}\right)=(\mathcal{F} T)\left(|x|^{2}+|y|^{2}\right)
$$

where $T=(2 \pi)^{-1} \rho^{2(1-n)} \mathrm{e}^{\mathrm{i} \rho^{2} t} \in \mathcal{D}_{L^{1},-n}^{\prime}(\mathbb{R})$. Hence, if $\alpha \in \mathbb{N}_{0}^{n}$ and $|\alpha|=j$, then formula (6.7) furnishes the following: 


$$
\begin{aligned}
\lambda_{\alpha} & =\frac{\rho^{2(1-n)}}{2 \pi} \int_{-\infty}^{\infty} \frac{(1-\mathrm{i} t)^{j}}{(1+\mathrm{i} t)^{j+n}} \mathrm{e}^{\mathrm{i} \rho^{2} t} \mathrm{~d} t=\mathrm{i} \rho^{2(1-n)} \operatorname{Res}_{t=\mathrm{i}}\left[\mathrm{e}^{\mathrm{i} \rho^{2} t} \frac{(1-\mathrm{i} t)^{j}}{(1+\mathrm{i} t)^{j+n}}\right] \\
& =\mathrm{i} \rho^{2(1-n)} \mathrm{e}^{-\rho^{2}} \operatorname{Res}_{s=0}\left[\mathrm{e}^{\mathrm{i} \rho^{2} s} \frac{(-\mathrm{i})^{j}(s+2 \mathrm{i})^{j}}{(\mathrm{i} s)^{j+n}}\right] \\
& =(-1)^{j} \mathrm{e}^{-\rho^{2}} \operatorname{Res}_{s=0}\left[\sum_{k=0}^{j} \sum_{l=0}^{\infty}\left(\begin{array}{l}
j \\
k
\end{array}\right)(2 \mathrm{i})^{k} \frac{\left(\mathrm{i} \rho^{2}\right)^{l+1-n}}{l !} s^{l-k-n}\right] \\
& =(-1)^{j} \mathrm{e}^{-\rho^{2}} \sum_{k=0}^{j} \frac{\left(-2 \rho^{2}\right)^{k}}{(k+n-1) !}\left(\begin{array}{l}
j \\
k
\end{array}\right) \\
& =\frac{(-1)^{j} j !}{(j+n-1) !} \mathrm{e}^{-\rho^{2}} L_{j}^{n-1}\left(2 \rho^{2}\right)=\frac{(-1)^{j} j !}{(j+n-1) !}\left\langle L_{j}^{n-1}(2 t) \mathrm{e}^{-t}, S\right\rangle,
\end{aligned}
$$

compare [20, Equ. (20.2), p. 95] or [5, Equ. 8.970.1] for the last line. This completes the proof.

Remark For $\sigma=(\mathcal{F} T)\left(|x|^{2}+|y|^{2}\right) \in \mathcal{S}^{\prime}\left(\mathbb{R}^{2 n}\right)$, we can conclude from the corollary to Proposition 5, in the same way as in the one-dimensional case, that $W_{\sigma} \in \mathcal{L}\left(L^{2}\left(\mathbb{R}^{n}\right)\right)$ if $T \in\left(1+t^{2}\right)^{n / 2} \cdot \mathcal{M}^{1}(\mathbb{R})$ and that $W_{\sigma} \in \operatorname{Com}\left(L^{2}\left(\mathbb{R}^{n}\right)\right)$ if $T \in\left(1+t^{2}\right)^{n / 2} \cdot L^{1}(\mathbb{R})$.

Examples If $n \geq 2$ and

$$
\sigma(x, y)=\delta\left(\rho-\sqrt{|x|^{2}+|y|^{2}}\right)=2 \rho \delta\left(\rho^{2}-|x|^{2}-|y|^{2}\right), \quad \rho>0
$$

then $\sigma=(\mathcal{F} T)\left(|x|^{2}+|y|^{2}\right)$ with $T(t)=\frac{\rho}{\pi} \mathrm{e}^{\mathrm{i} \rho^{2} t} \in\left(1+t^{2}\right)^{n / 2} L^{1}(\mathbb{R})$ and hence $W_{\sigma} \in$ $\operatorname{Com}\left(L^{2}\left(\mathbb{R}^{n}\right)\right)$ by the above remark. More precisely, we have $\sigma=\Phi_{r}\left(2 \rho^{2 n-1} \delta_{\rho^{2}}\right)$ and therefore $W_{\sigma} h_{\alpha}=\lambda_{|\alpha|} h_{\alpha}$ where

$\lambda_{j}=\frac{2(-1)^{j} j ! \rho^{2 n-1}}{(j+n-1) !} L_{j}^{n-1}\left(2 \rho^{2}\right) \mathrm{e}^{-\rho^{2}} \sim \frac{2(-1)^{j} \rho^{n-1 / 2}}{\sqrt{\pi}(2 j)^{(2 n-1) / 4}} \cos \left(2 \rho \sqrt{2 j}-\frac{(2 n-3) \pi}{4}\right)$

for $j \rightarrow \infty$, see [5, Equ. 8.978.3]. The kernel of the pseudo-differential operator $W_{\sigma}$ is given by

$$
\begin{aligned}
W_{\sigma}(x, y)= & \frac{\rho Y(2 \rho-|x+y|)}{(2 \pi)^{n / 2}} \\
& \cdot \frac{\left(\rho^{2}-|x+y|^{2} / 4\right)^{n / 4-1 / 2}}{|x-y|^{n / 2-1}} J_{n / 2-1}\left(|x-y| \sqrt{\rho^{2}-\frac{|x+y|^{2}}{4}}\right) .
\end{aligned}
$$

Let us finally mention that the calculation of the "oscillator semigroup", cf. [3, Ch. 5, p. 223], becomes deceptively easy upon employing formula (6.7). In fact, for

$$
\sigma_{\zeta}(x, y)=\exp \left(-\zeta\left(|x|^{2}+|y|^{2}\right)\right), \quad(x, y) \in \mathbb{R}^{2 n}, \zeta \in \mathbb{C}, \operatorname{Re} \zeta \geq 0,
$$


we have $\sigma_{\zeta}=\left(\mathcal{F} T_{\zeta}\right)\left(|x|^{2}+|y|^{2}\right)$ with

$$
T_{\zeta}=\left\{\begin{array}{ll}
\frac{\zeta}{\pi\left(\zeta^{2}+t^{2}\right)} & : \zeta \neq 0, \\
\delta & : \zeta=0
\end{array}\right\} \in \mathcal{D}_{L^{1},-n}^{\prime}(\mathbb{R})
$$

and hence $W_{\sigma_{\zeta}} h_{\alpha}=\lambda_{|\alpha|}(\zeta) h_{\alpha}, \alpha \in \mathbb{N}_{0}^{n}$, where (for $\operatorname{Re} \zeta \geq 0, \zeta \neq 0, j \in \mathbb{N}_{0}$ )

$$
\begin{aligned}
\lambda_{j}(\zeta) & =\int_{-\infty}^{\infty} \frac{(1-\mathrm{i} t)^{j}}{(1+\mathrm{i} t)^{j+n}} \cdot \frac{\zeta}{\pi\left(\zeta^{2}+t^{2}\right)} \mathrm{d} t \\
& =-2 \mathrm{i} \zeta \operatorname{Res}_{t=-\mathrm{i} \zeta}\left(\frac{(1-\mathrm{i} t)^{j}}{(1+\mathrm{i} t)^{j+n}\left(\zeta^{2}+t^{2}\right)}\right)=\frac{(1-\zeta)^{j}}{(1+\zeta)^{j+n}}
\end{aligned}
$$

in accordance with [3, Equ. (5.30), p. 238].

Acknowledgements Open access funding provided by University of Innsbruck and Medical University of Innsbruck.

Open Access This article is distributed under the terms of the Creative Commons Attribution 4.0 International License (http://creativecommons.org/licenses/by/4.0/), which permits unrestricted use, distribution, and reproduction in any medium, provided you give appropriate credit to the original author(s) and the source, provide a link to the Creative Commons license, and indicate if changes were made.

\section{References}

1. Brychkov, Yu.A., Marichev, O.I., Prudnikov, A.P.: Integrals and Series. Vol. 2 (Special Functions), Gordon \& Breach, New York (1986); Transl from А. П. Прудников, Ю. А. Брычков и О.И. Маричев: Интегралы и Ряды, Специальные Функции, Наука, Москва (1983)

2. Brychkov, Yu.A., Marichev, O.I., Prudnikov, A.P. : Integrals and series. Vol. 3 (More Special Functions). Gordon \& Breach, New York (1990); Transl. from А. П. П рудников, Ю. А. Брычков и О.И. Маричев: Интегралы и Ряды, Дополнительные Главы, Наука, Москва (1986)

3. Folland, G.B.: Harmonic Analysis in Phase Space, Annals of Math. Studies 122. Princeton Univ. Press, Princeton (1989)

4. Friedlander, G., Joshi, M.: Introduction to the Theory of Distributions, 2nd edn. Cambridge Univ. Press, Cambridge (1998)

5. Gradshteyn, I.S., Ryzhik, I.M. : Table of Integrals, Series and Products, Academic Press, New York (1980); Transl. from И.С. Градштейн и И.М. Рыжик: Таблицы Интегралов, Сумм, Рядов и Произведений, Наука, Москва (1971)

6. Horváth, J.: Topological Vector Spaces and Distributions, vol. I. Addison-Wesley, Reading, MA (1966)

7. Ortner, N., Wagner, P.: Applications of weighted $\mathcal{D}_{L}^{\prime}{ }^{p}$-spaces to the convolution of distributions. Bull. Polish Acad. Sci. Math. 37, 579-595 (1989)

8. Ortner, N., Wagner, P.: Distribution-Valued Analytic Functions. Tredition, Hamburg (2013)

9. Ortner, N., Wagner, P.: Fundamental Solutions of Linear Partial Differential Operators. Springer, New York (2015)

10. Ortner, N., Wagner, P.: Fourier transformation of $\mathrm{O}(\mathrm{p}, \mathrm{q})$-invariant distributions. Fundamental solutions of ultra-hyperbolic operators. J. Math. Anal. Appl. 450, 262-292 (2017)

11. Reed, M., Simon, B.: Methods of Modern Mathematical Physics. Functional Analysis, vol. 1. Academic Press, New York (1980). (Revised ed.)

12. Schwartz, L.: Les opérateurs invariants par rotation, l'opérateur $\Delta$, Sém. Schwartz: Équations aux Dérivées Partielles, Exp. 7. Fac. Sci. Paris (1954/1955)

13. Schwartz, L.: Causalité et analyticité, Sém. Schwartz-Lévy: Mathématiques et Théorie Quantique des Champs, Exp. 3. Fac. Sci. Paris (1956/1957): published in Anais Acad. Bras. Ci. 34, 13-21 (1962) 
14. Schwartz, L.: Théorie des distributions à valeurs vectorielles. I. Ann. Inst. Fourier 7, 1-141 (1957)

15. Schwartz, L.: Théorie des Distributions. Nouv. éd., Hermann, Paris (1966)

16. Simon, B.: The Weyl transform and $L^{p}$ functions on phase space. Proc. Am. Mat. Soc. 116, 1045-1047 (1992)

17. Thangavelu, S.: Lectures on Hermite and Laguerre Expansions, Math. Notes 42. Princeton University Press, Princeton (1993)

18. Treves, F.: Linear Partial Differential Equations with Constant Coefficients. Gordon and Breach, New York (1967)

19. Wagner, E.: Asymptotische Darstellungen der hypergeometrischen Funktionen für große Werte eines Parameters. Zeitschrift Anal. Anw. 1, 1-11 (1982)

20. Wong, M.W.: Weyl Transforms. Springer, New York (1998)

Publisher's Note Springer Nature remains neutral with regard to jurisdictional claims in published maps and institutional affiliations. 